\title{
Multi-axial creep-fatigue life prediction considering history- dependent damage evolution: A new numerical procedure and experimental validation
}

Run-Zi Wang ${ }^{\text {a }}$, Su-Juan Guo ${ }^{\text {a,* }}$, Haofeng Chen ${ }^{\mathrm{b}}$, Jian-Feng Wen ${ }^{\text {a }}$, Xian-Cheng Zhang $^{\text {a,* }}$, Shan-Tung Tu ${ }^{\text {a }}$

${ }^{a}$ Key Laboratory of Pressure Systems and Safety, Ministry of Education, East China University of Science and Technology, Shanghai 200237, P.R.China

${ }^{\mathrm{b}}$ Department of Mechanical \& Aerospace Engineering, University of Strathclyde, Glasgow, G1 1XJ, UK

\begin{abstract}
In this paper, a new numerical procedure based on a cycle-by-cycle analysis has been constructed for creep-fatigue behavior and life prediction of high-temperature structures under multi-axial stress states. Within this framework, a modified unified viscoplastic constitutive model with isotropic hardening and modified kinematic hardening rules is developed to simulate the cycle-by-cycle stress-strain responses.
\end{abstract}

${ }^{*}$ Corresponding author: Key Laboratory of Pressure Systems and Safety, Ministry of Education, School of Mechanical and Power Engineering, East China University of Science and Technology, Meilong Road 130, Xuhui District, Shanghai, 200237, P.R. China. Tel.: +862164253513.

E-mail address: sujuanguo@ecust.edu.cn(S.J. Guo), xczhang@ecust.edu.cn_(X.C. Zhang) 
Moreover, the newly constructed creep-fatigue approach calculates fatigue and creep damage variables using the critical plane method (CPM) and the modified strain energy density exhaustion (SEDE) model, respectively. The multi-axial ductility factor and elastic follow-up factor are also introduced into the modified SEDE model to accommodate the special multi-axial and mixed controlled modes, which are widely existed in practical structures. In order to validate the feasibility of the proposed numerical procedure, a series of creep-fatigue tests of notched specimens made from nickel-based GH4169 superalloy were carried out at $650{ }^{\circ} \mathrm{C}$. The predicted numbers of cycles to crack initiation agree well with the experimental data. Evidence of crack initiation under various loading conditions was observed via the electron backscatter diffraction (EBSD) technique, indicating location-dependent crack initiations depending on loading conditions. In detail, the crack initiation sites shifting from surface to subsurface with increasing hold times can be well simulated by the proposed numerical procedure due to a reasonable description of the creepfatigue damage evolution.

Keywords: Creep-fatigue; Unified viscoplastic model; Life prediction; Crack initiation sites; Multi-axial stress states

\section{Introduction}

With the demand for higher performance of aero engines, the safety assessments of critical components such as turbine disks operated at elevated temperatures under 
cyclic service loadings are of great significance to their structural integrity (Pineau et al., 2016). Under such circumstances, fatigue induced by frequent start-ups and shutdowns and creep induced by steady hold time periods together is very likely to cause the crack initiation in some critical positions of a turbine disk. The material degradation in the structures is greatly affected by the time-dependent fatigue, creep and creep-fatigue interactions (Ahmed et al., 2016). In particular, some high temperature components have been found to exhibit distinct damage redistributions with mutative maximum locations under the multi-axial creep-fatigue loading conditions throughout the lifetime (Wang et al., 2012). An accurate description of history-dependent damage at critical locations of high temperature components is essential for the development of a robust design methodology and reliable life prediction techniques. As the computer technology develops, the finite element method (FEM) provides an effective tool for obtaining the cycle-by-cycle stressstrain behavior as well as the accurate history-dependent damage estimations for complex structures. Several key factors dictating an accurate creep-fatigue life design should be essentially considered by taking advantage of the FEM. A reasonable cyclic constitutive model directly coupled with the FEM is of necessity to simulate the parallel evolution of deformation of a component under loading, hold time and unloading periods. Then, some reliable life models with clear physical meanings should be selected to estimate different types of damage. In addition to this, the multi-axial stress states caused by geometric discontinuities and complicated loading paths should be incorporated for the physics of crack initiation (Yang et al., 
2015).

In the past several decades, many researchers have devoted themselves to the development of creep-fatigue life models in terms of varied theoretical systems. The strategies for creep-fatigue crack initiation life prediction can be classified into microscopic and macroscopic groups. For the microstructure-based approaches, fatigue indicator parameters, such as accumulated plastic slips and stored dissipation energy, can be applied to characterizing the fatigue damage evolution process (Dunne, 2014; Sangid, 2013), while the grain boundary cavitation due to grain boundary sliding is taken as an indicator contributing to the creep damage evolution process (Wen et al., 2017). Furthermore, the dislocation-mechanics cyclic viscoplasticity model enjoys the great advantage in revealing micro-mechanisms of strengthening and softening for high-temperature deformation, where a key variable regarding dislocation density can be used to characterize the precipitate and grain boundary strengthening, low-angle boundary dislocation annihilation and so on (Barrett et al., 2017; Barrett et al., 2014b). However, although the physical process of crack initiation can be well revealed with the microstructure-based approaches, it is difficult for these approaches to meet the requirement of crack initiation life prediction in the component-level analysis. Hence, macroscopic phenomenological approaches are widely employed in the current assessments and design codes (Ainsworth, 2003; ASME, 2009; RCC-MRx, 2012) due to their simple implementation and low computational consumption. In the creep-fatigue regime, a modeling analysis dealing with fatigue or creep loading conditions separately is not 
adequate for safety and reliability of design (Naumenko and Altenbach, 2007; Socie and Marquis, 2000). Therefore, the linear damage summation (LDS) has been widely accepted to predict the creep-fatigue crack initiation life. Traditionally, fatigue damage was estimated using the phenomenological fatigue curves obtained from pure fatigue tests based on the cycle fraction concept (Manson, 1968). Much attention was paid to the estimation of creep damage, including the stress-based time fraction approach (Robinson, 1952), the strain-based ductility exhaustion approach (Hales, 1980; Priest and Ellison, 1981) and their extended ones (Spindler, 2005; Spindler and Payten, 2011; Takahashi et al., 2009). The above-mentioned approaches are based on the steady stress-strain response, lacking the description of real-time damage evolution. Recently, the strain energy density exhaustion (SEDE) method based on a cycle-by-cycle concept has been proved to be capable of improving the prediction capacity (Wang et al., 2017b). Its advantages are two-fold. One is that the concept of energy dissipation criterion (EDC) is introduced to fulfill the physical meaning of this approach. The other is that key experimental variables in each cycle are extracted to calculate cycle-dependent damage parameters whereby cyclic plastic behavior is taken into account naturally. However, the cycle-by-cycle creep-fatigue life model has just been applied in the uniform stress field without any geometric discontinuities (Wang et al., 2017b). Aiming at practical high temperature structures, the SEDE model will have to be modified by considering the influence of multi-axial stress states, which have a great impact on the fatigue and creep damage calculations in stress concentration regions (Fatemi and Socie, 1988; Wen et al., 2016). Another 
important issue concerning actual high temperature structures is that a ubiquitous mixed controlled mode during hold time periods, namely an intermediate mode between the stress-controlled and the strain-controlled modes (Wang et al., 2018b). In this case, the elastic follow-up effect should be taken into account with respect to the modified SEDE model. Furthermore, accurate history-dependent stress and strain fields in each position of a high temperature structure are critical in terms of the accurate implementation of the cycle-by-cycle modified SEDE damage model. In order to achieve this goal, a reasonable constitutive equation is required for creepfatigue behavior simulations. In this regard, the unified viscoplastic constitutive models with the static recovery term (Chaboche, 2008; Yaguchi et al., 2002; Zhang and Xuan, 2017), which can well reflect the interaction between creep and fatigue, have been widely accepted among numerous constitutive models (such as the nonunified constitutive equations, etc (Chen et al., 2017; Kobayashi et al., 2003)). It is found that the stress relaxation evolutionary rule of the object material GH4169 superalloy in this work exhibits obvious loading level dependence at $650{ }^{\circ} \mathrm{C}$. Nevertheless, the influence of loading levels (i.e., plastic strain amplitude) on the creep or stress relaxation evolutionary rule has not been considered in the existing works.

In this work a new unified viscoplastic constitutive model (NUVCM) was developed at first by introducing a modified static recovery term in the kinematic hardening rule considering the effect of loading levels for the GH4169 superalloy at $650{ }^{\circ} \mathrm{C}$. Then, the fatigue and creep damage models were respectively presented by 
employing the concept of "critical plane" (CPM) (Findley, 1958) as well as multiaxial ductility factor (Wen et al., 2016), with the purpose of estimating multi-axial fatigue damage and multi-axial creep damage, respectively. Also, an elastic followup factor was introduced in the current creep damage model for the special mixed controlled mode in structures. Finally, cyclic tests on single-edge-notch specimens were carried out to validate the feasibility and robustness of the proposed numerical procedure from both experimental and simulative viewpoints, during which the physical details of the creep-fatigue crack initiations of the single-edge-notch specimens are successfully revealed.

\section{Experimental procedures}

In order to validate the applicability of the proposed multi-axial creep-fatigue life prediction procedure, a series of fatigue and creep-fatigue tests were carried out at $650{ }^{\circ} \mathrm{C}$ for uniaxial uniform and notched specimens made from nickel-based GH4169 superalloy. After that, metallographic observations were performed through the electron backscatter diffraction (EBSD) technique to characterize crack initiation sites of the notched specimens undergoing various loading conditions.

\subsection{Preparation of specimens}

The chemical compositions of the nickel-based GH4169 superalloy are shown in Table 1. For the creep-fatigue tests, the preparation processes of specimens are schematically shown in Fig. 1. Firstly, rough machining cylindrical bars were extracted from the as-received disk using the electrical discharge machining wire 
cutting (wire EDM). Then, a standard heat treatment (HT) was carried out to ensure the optimized distributions of $\mathrm{Ni}_{3} \mathrm{Al}$ type $\gamma^{\prime}$ and $\mathrm{Ni}_{3} \mathrm{Nb}$ type $\gamma^{\prime \prime}$ strengthening phases. The detail of the standard HT is listed as follows: solid solution at $960{ }^{\circ} \mathrm{C}$ for $60 \mathrm{~min}$, air cooling (AC) to room temperature (RT), aging at $720^{\circ} \mathrm{C}$ for $480 \mathrm{~min}$, furnace cooling to $620{ }^{\circ} \mathrm{C}$ for $120 \mathrm{~min}$, aging again at $620{ }^{\circ} \mathrm{C}$ for another $480 \mathrm{~min}$, and then AC to RT. Finally, the uniaxial uniform and notched specimens were machined from the above-mentioned heat treated specimens (HTS), the detailed dimensions of which are shown in Fig. $2 \mathrm{a}$ and $2 \mathrm{~b}$, respectively. The uniaxial uniform specimens were fine machined from the HTS, in which the gauge length was polished using DiaPro Dac diamond suspension. For each notched specimen, a single edge notch with a radius of $8 \mathrm{~mm}$ was located at the center of the gauge portion by the additional wire EDM method after fine machining. The critical position of notch surface was polished up to a mirror surface to avoid the local stress concentration at the notch root.

\subsection{Fatigue and creep-fatigue tests}

Strain-controlled fatigue and creep-fatigue tests were carried out at $650{ }^{\circ} \mathrm{C}$ using a MTS model $809 \mathrm{~A} / \mathrm{T}$ testing system. The input parameters for the uniaxial and notched specimens regarding the strain-controlled tests are summarized in Table 2. Strain-controlled triangular loading waveforms were employed for pure fatigue tests, while tension-hold-only trapezoidal loading waveforms were employed for creepfatigue tests. Specimens U-1 to U-4 denote the uniaxial uniform specimens (Fig. 2a), 
which are used to determine the material parameters for the NUVCM. Detailed experimental procedures and loading conditions for the uniaxial uniform specimens have been reported in our previous work (Wang et al., 2017b). Specimens N-1 to N12 represent the notched specimens (Fig. 2b). The aims of this group are to prove the feasibility of the proposed numerical procedure in multi-axial creep-fatigue life prediction and to discuss the crack initiation mechanisms. Testing temperature, $T$, strain ratio, $R_{\varepsilon}$, and strain rate, $\dot{\varepsilon}$, were respectively set to be $650{ }^{\circ} \mathrm{C},-1$ and $0.4 \% / \mathrm{s}$, which were the same as those for the uniaxial uniform specimens. Note that specimens $\mathrm{N}-1$ to $\mathrm{N}-12$ were subjected to global strain-controlled loading waveforms with a total strain range, $\Delta \varepsilon_{t}$, ranging from $0.6 \%$ to $1.0 \%$, as shown in Fig. 3. Crack initiation life of all the specimens are summarized in the last column of Table 2. It is also shown that two ceramic rods of a high temperature extensometer were symmetrically attached to the gauge-length area of the specimen. The gap between the two ceramic rods was calibrated to be $25 \mathrm{~mm}$ before clamping both sides of each specimen. Hold times at the peak tensile strain period, $t_{h}$, for each $\Delta \varepsilon_{t}$ were selected to be $0 \mathrm{~s}, 60 \mathrm{~s}, 600 \mathrm{~s}$ and $3600 \mathrm{~s}$. The replica technique (Jordon et al., 2012) based on a dispensing gun with a mixing nozzle was adopted to detect crack initiation length at the notch surface, and the crack initiation life for each notched specimen is determined when the main crack at the notch root reached to a critical value of $0.3 \mathrm{~mm}$ (Meyer-Olbersleben et al., 1999).

\subsection{Metallographic observations for post-test examination}


In order to study the crack initiation mechanisms of the notched specimens undergoing differing loading conditions, post-test examinations on creep-fatigue tests were conducted using the EBSD technique. After determining the crack initiation life of each notched specimen, the longitudinal cross section near the notch root was prepared by the wire EDM. With a combination of scanning electron microscopy (SEM) and high quality EBSD Kikuchi patterns, the crack initiation sites were captured by a CamScan Apollo 300 SEM equipped with a Hikari EBSD detector. The key factor for ensuring a high-resolution EBSD map is to remove residual stress as far as possible. To this end, a careful treatment was conducted as follows: SiC Foil \#220 paper at $25 \mathrm{~N}$ force for $1 \mathrm{~min}$ with water-based diamond suspension, MD-Largo polishing disc at $25 \mathrm{~N}$ force for $4 \mathrm{~min}$ with DiaPro Allegro/Largo diamond suspension, MD-Dac polishing disc at $20 \mathrm{~N}$ force for $3 \mathrm{~min}$ with DiaPro Dac diamond suspension, and finally MD-Chem polishing disc at $15 \mathrm{~N}$ force for $15 \mathrm{~min}$ with oxide polishing suspension (OPS). After the sample preparation, Kikuchi patterns were collected by the Hikari detector and EBSD data were processed by the Oxford Instruments HKL Channel 5 and the Tango software.

\section{The proposed numerical procedure}

\subsection{Overview}

The overall flow chart of the proposed numerical procedure for predicting creepfatigue life of high temperature structures is shown in Fig. 4. The first task for the proposed numerical procedure was to develop the NUVCM whereby creep-fatigue 
behavior of GH4169 superalloy at $650{ }^{\circ} \mathrm{C}$ can be well described. After that, the NUVCM can be implemented into the finite element code ABAQUS using user material subroutine (umat). The second task was to simulate the cyclic deformation process of the notched specimens under creep-fatigue loadings, during which the cycle-by-cycle stress and strain evolution fields of all the nodes in the notched specimens can be obtained. The third task was to extract the element integration points information (i.e., equivalent stress, equivalent plastic strain etc.) and to derive the fatigue and creep damage variables distributions by the usdfld user subroutine. In this period, the uniaxial cycle-by-cycle SEDE model developed in the previous work (Wang et al., 2017b) was further modified with the purpose of incorporating the multi-axial stress states and elastic follow-up effect. In detail, a modified fatigue damage parameter based on the CPM was elaborated to estimate the fatigue damage in the $i$ th cycle, $d_{f}^{(i)}$, while a modified creep damage parameter obtained by employing the additional factors was utilized to estimate creep damage in the $i$ th cycle, $d_{c}^{(i)}$. Finally, the cycle-dependent fatigue and creep damages were recorded through the usdfld subroutine. With the aid of LDS rule, if the summation of accumulated fatigue and creep damages up to $j$ cycle, $\sum_{i=1}^{j} d_{f}^{(i)}+d_{c}^{(i)}$, equals to 1 at the most dangerous integration point, the final creep-fatigue crack initiation life can be determined. Considering that the effect of accumulated damage on stress-strain behavior is limited within the crack initiation period, the creep-fatigue deformation and creep-fatigue damage were independently described in the proposed numerical procedure. The corresponding NUVCM and creep-fatigue damage models will be 
introduced in the following paragraphs.

\subsection{Development of the NUVCM}

The NUVCM involving modified Chaboche's nonlinear kinematic hardening rule and the isotropic hardening rule was developed in this section to describe the cyclic deformation and stress relaxation behavior under creep-fatigue loading conditions. Meanwhile, the small deformation and initial isotropic elasticity are assumed here.

\subsubsection{Main constitutive equations}

The main equations of the unified constitutive model developed in this paper are written as follows

$$
\begin{gathered}
\boldsymbol{\varepsilon}^{t}=\boldsymbol{\varepsilon}^{e}+\boldsymbol{\varepsilon}^{i n} \\
\boldsymbol{\varepsilon}^{e}=\frac{1+v}{E} \boldsymbol{\sigma}-\frac{v}{E}(\operatorname{tr} \boldsymbol{\sigma}) \mathbf{I} \\
\dot{\boldsymbol{\varepsilon}}^{i n}=\frac{3}{2} \dot{p} \frac{\mathbf{s}-\boldsymbol{\alpha}}{\|\mathbf{s}-\boldsymbol{\alpha}\|} \\
\dot{p}=\sqrt{\frac{2}{3} \dot{\boldsymbol{\varepsilon}}^{i n}: \dot{\boldsymbol{\varepsilon}}^{i n}}=\left\langle\frac{F}{K}\right\rangle^{n} \\
F=\sqrt{\frac{3}{2}(\mathbf{s}-\boldsymbol{\alpha}):(\mathbf{s}-\boldsymbol{\alpha})}-Q_{0}-R
\end{gathered}
$$

where the total strain $\boldsymbol{\varepsilon}^{t}$ is separated into the elastic component $\boldsymbol{\varepsilon}^{e}$ and inelastic component $\varepsilon^{i n}$, and the creep and plastic deformation are treated together as a uniform inelastic variable. Meanwhile, the linear elasticity obeys the Hooke's law as Eq. (2), in which $E$ and $v$ denote Young's modulus and Poisson's ratio, respectively. $\boldsymbol{\sigma}$ and tro are the stress tensor and the trace of stress tensor, and $\mathbf{I}$ is the unit tensor 
of second-rank. In addition, $\dot{\boldsymbol{\varepsilon}}^{i n}$ and $\dot{p}$ are the rate of inelastic strain and accumulated inelastic strain, respectively. $\mathbf{s}$ and $\boldsymbol{\alpha}$ are the deviators of stress tensor and back stress tensor, respectively. $F$ is the von-Mises yield function, $K$ and $n$ are the material parameters representing the viscous characteristics of the investigated material. $Q_{0}$ is the initial yield stress, and $R$ is isotropic deformation resistance reflecting the change of the yield surface size. (:) represents the inner product between second-rank tensors, and \langle\rangle is the MaCauley bracket, which means that $\langle x\rangle=0$ when $\mathrm{x}<0$ and $\langle x\rangle=x$ when $x \geq 0$. It should be mentioned that some researchers adopted the hyperbolic sine equation to describe the inelastic strain rates, instead of power-law equation of Eq. (4). Both have their advantages and limitations. For traditional power-law equation, the main advantage is that when the value of $n$ is high enough, it can also describe rate-independent cyclic behavior. Moreover, the procedure to derive the consistency tangent modulus will be quite simple if the power-law equation is adopted, indicating good applicability of Eq. (4). Barrett et al (Barrett et al., 2014a; Barrett et al., 2013a; Barrett et al., 2013b) pointed out that a hyperbolic sine equation enables reliable extrapolation from strain-rates at which experiments are conducted to strain-rates typically observed in practical applications. In addition, the limit viscosity influence under the monotonic tensile loadings and strain rate jump influence under some extreme cyclic loadings can be well depicted when a hyperbolic sine equation is used (Chen and Feng, 2015; Nouailhas, 1989).

\subsubsection{Development of the kinematic hardening rule}

As reported by Kang and Kan (Kang and Kan, 2007), Yaguchi and Takahashi 
(Yaguchi et al., 2002), Zhang and Xuan (Zhang and Xuan, 2017), Wang and Shi (Wang et al., 2017a), etc., the introduction of the static recovery term in the kinematic hardening rule can effectively improve the capability of the unified viscoplastic constitutive model. In particular, it can be used to simulate the creepfatigue interaction and the creep/relaxation behavior during a stress or strain hold time for materials under creep-fatigue loadings. A modified Chaboche's nonlinear kinematic hardening rule is developed in this paper by introducing a new static recovery term, in which the maximum plastic strain amplitude is involved to reflect the loading level influence. Different from the classic Choboche's model, the total back stress is assumed to divided into 8 parts in this paper for a better nonlinearity, namely $\boldsymbol{\alpha}=\sum_{i=1}^{8} \boldsymbol{\alpha}_{i}$. The evolution equation for each back stress part $\boldsymbol{\alpha}_{i}$ is set as:

$$
\begin{gathered}
\dot{\boldsymbol{\alpha}}_{i}=\zeta_{i}\left(\frac{2}{3} r_{i} \dot{\boldsymbol{\varepsilon}}^{i n}-\boldsymbol{\alpha}_{i} \dot{p}\right)-\gamma[\bar{\alpha}]^{m(q)} \boldsymbol{\alpha}_{i} \\
m(q)=\phi_{1} e^{-q / \omega}+\phi_{2}
\end{gathered}
$$

where $\zeta_{i}$ and $r_{i}$ are the material parameters for each part of back stress, $\bar{\alpha}=\left(\frac{3}{2} \alpha_{i}: \alpha_{i}\right)^{1 / 2}$ is the equivalent back stress. $\gamma$ and $m$ are two conventional parameters controlling the magnitude of static recovery and are always assumed to be identical for each part of back stress (Zhang and Xuan, 2017). In order to describe the stress relaxation behavior reasonably under different loading levels, the parameter $m(q)$ is redefined as an exponential function of plastic strain amplitude $q$ as shown in Eq. (7). $\phi_{1}, \phi_{2}$ and $\omega$ are three temperature-dependent parameters which can be determined by creep-fatigue experiments under different loading levels. 
The maximum plastic strain amplitude can be obtained by the plastic strain memorization method which comes from Chaboche's previous work (Chaboche et al., 1979), and can be expressed as

$$
\begin{gathered}
f=\frac{2}{3}\left(\boldsymbol{\varepsilon}^{p}-\boldsymbol{\beta}^{p}\right):\left(\boldsymbol{\varepsilon}^{p}-\boldsymbol{\beta}^{p}\right)-q^{2} \leq 0 \\
\boldsymbol{\beta}^{p}=(1-\eta) H(f)\left(\dot{\boldsymbol{\varepsilon}}^{p}: \mathbf{n}^{\prime}\right): \mathbf{n}^{\prime} \\
\dot{q}=\eta H(f)\left\langle\mathbf{n}: \mathbf{n}^{\prime}\right\rangle \dot{p}
\end{gathered}
$$

where $\mathbf{n}^{\prime}=\left(\partial f / \partial \boldsymbol{\varepsilon}^{p}\right) /\left\|\partial f / \partial \boldsymbol{\varepsilon}^{p}\right\|, \mathbf{n}=\dot{\boldsymbol{\varepsilon}}^{p} /\left\|\dot{\boldsymbol{\varepsilon}}^{p}\right\| . f$ is the strain memory surface, $\boldsymbol{\beta}^{p}$ and $q$ represent the center and radius of the surface, and $\eta$ is the percentage between the isotropic and the kinematic part in the NUVCM. Note that the saturated value of $q$ denotes the maximum plastic strain amplitude of the current loading condition.

\subsubsection{Development of the isotropic hardening rule}

The isotropic hardening rule combined with a linear term (Lemaitre and Chaboche, 1990) is employed to express the specific feature of cyclic softening for the nickelbased GH4169 superalloy. The scalar isotropic hardening variable is a function of accumulated inelastic strain and takes the form

$$
\dot{R}=b\left(Q_{s a}-R\right) \dot{p}+H(1+b p) \dot{p}
$$

where $Q_{s a}$ is the asymptotic value of the isotropic deformation resistance in the first stage of rapid softening, $b$ is the speed approaching to the asymptotic value in the first stage, and $H$ is associated with the slope in the second stage of linear softening.

\subsection{Energy-based creep-fatigue damage models}

\subsubsection{Energy dissipation criterion}


The physical basis of the energy-based damage models, in particular for the description of creep damage, follows the energy dissipation criterion (Chen et al., 2007; Fan et al., 2015). In a thermodynamic system, a deforming specimen follows the energy conservation principle, i.e.,

$$
\frac{\mathrm{d} U}{\mathrm{~d} t}+\frac{\mathrm{d} K}{\mathrm{~d} t}=\frac{\mathrm{d} W}{\mathrm{~d} t}+\frac{\mathrm{d} \Theta}{\mathrm{d} t}
$$

where $U$ is the internal energy, $K$ is the kinetic energy in the macroscopic view, $W$ is the mechanical work, and $\Theta$ is the thermal energy. Each term in the above equation can be further written as

$$
\begin{gathered}
\frac{\mathrm{d} U}{\mathrm{~d} t}=\int_{V} \rho \frac{\mathrm{d} e}{\mathrm{~d} t} \mathrm{~d} V \\
\frac{\mathrm{d} K}{\mathrm{~d} t}=\int_{V} \rho \boldsymbol{v} \cdot \frac{\mathrm{d} \boldsymbol{v}}{\mathrm{d} t} \mathrm{~d} V \\
\frac{\mathrm{d} W}{\mathrm{~d} t}=\int_{V}\left(\rho \boldsymbol{v} \frac{\mathrm{d} \boldsymbol{v}}{\mathrm{d} t}+\boldsymbol{\sigma} \frac{\mathrm{d} \boldsymbol{\varepsilon}}{\mathrm{d} t}\right) \mathrm{d} V \\
\frac{\mathrm{d} \Theta}{\mathrm{d} t}=\int_{V}\left(-\frac{\partial \boldsymbol{q}}{\partial \boldsymbol{x}}+\rho r\right) \mathrm{d} V
\end{gathered}
$$

where $V$ is the volume, $v$ is the velocity, $\rho$ is the density, $e$ is the internal energy per unit mass, $\boldsymbol{q}$ is the heat flux across a normal unit area, and $r$ is the heat supply per unit mass.

Combined Eq. (12) with (13a-d), a partial expression can be obtained, i.e.,

$$
\rho \frac{\mathrm{d} e}{\mathrm{~d} t}-\boldsymbol{\sigma} \frac{\mathrm{d} \boldsymbol{\varepsilon}}{\mathrm{d} t}+\left(\frac{\partial \boldsymbol{q}}{\partial \boldsymbol{x}}-\rho r\right)=0
$$

where $\rho \frac{\mathrm{d} e}{\mathrm{~d} t}$ is the change rate of internal energy per unit mass, $\boldsymbol{\sigma} \frac{\mathrm{d} \boldsymbol{\varepsilon}}{\mathrm{d} t}$ is the strain energy density per unit time caused by mechanical work, $\left(\frac{\partial \boldsymbol{q}}{\partial \boldsymbol{x}}-\rho r\right)$ is the change of heat. Furthermore, Eq. (14) can be discretized at a very small time increment, i.e., 


$$
\rho \delta e=\boldsymbol{\sigma} \delta \boldsymbol{\varepsilon}+\delta \Theta
$$

This equation shows that the accumulation of internal energy equals to the changes of strain energy density (caused by mechanical work) and heat transformation. For creep-fatigue conditions at a constant temperature, all the heat transformation comes from the mechanical work. As a consequence, the right side of Eq. (15) is expressed as a function of mechanical work and can be rewritten as

$$
\rho \delta e=f(\delta w)=f(\hbar) \cdot \delta w
$$

where $w$ is the strain energy density, which can be regarded as the area of the stressstrain curve in a creep-fatigue condition, and $f(\hbar)$ is the energy dissipation function. Integrating Eq. (16) from the initial time to the failure time and considering the repeating numbers of cycles during this time period, we can obtain a new expression

$$
\sum_{i=1}^{N_{c-f}} \rho \Delta e_{i}=f(\hbar) \cdot \sum_{i=1}^{N_{c-f}} \Delta w_{i}
$$

where $N_{c-f}$ is the number of cycles to failure in a creep-fatigue condition. The key point of energy dissipation criterion is to select a suitable dissipation function to assess the damage and to predict the creep-fatigue life.

\subsubsection{Fatigue damage based on CPM}

Although the notched specimens are subjected to global uniaxial external loadings (Table 2), the critical point at each notched root always experiences multi-axial loading conditions due to the discontinuous geometry and residual stress field (Yang et al., 2015). Thus, a good expression of the multi-axial damage mechanism regarding the pure fatigue failure is to develop a fatigue damage parameter in the framework of CPM. The CPM was firstly introduced to illustrate the roles of shear 
stress and normal stress on different fatigue failure types. Later, Fatami-Socie (Fatemi and Socie, 1988), Smith-Watson-Topper (Smith, 1970), Wang-Brown (Wang and Brown, 1993, 1996) and Yu-Zhu models (Yu et al., 2017a, b) were developed to estimate fatigue life based on CPM. The critical plane-based fatigue damage parameters have been proved to show better capabilities in fatigue damage estimations under multi-axial stress states than the extended uniaxial fatigue models (Socie and Marquis, 2000). In addition, it is also in accordance with the physical mechanism that fatigue cracks are inclined to initiate on the preferred planes.

The basic feature of CPM is to recognize the most severe fatigue damage on a determined critical plane (Bonnand et al., 2010). Detailed procedures for that are depicted herein. Since the stress and strain tensors can be calculated by FEA, normal and shear strain/stress components for each integration point on all the potential planes can be obtained according to the tensor rotation rule as a function of plane orientation. Then, by means of an efficient search algorithm (Susmel, 2010; Yu et al., 2017b), the critical plane, in which the fatigue damage parameter is maximized, can be determined among all the potential planes. Ince et al (Ince and Glinka, 2014) proposed a generalized strain amplitude (GSA) parameter that gains success in applying to the global uniaxial loading conditions for the pure fatigue life assessment. More recently, Yu et al (Yu et al., 2017a) developed a very simple and effective expression by considering the roles of plastic normal/shear strains based on GSA. Combining these two works (Ince and Glinka, 2014; Yu et al., 2017a), one obtains an equation relating to fatigue damage in a specific cycle, $d_{f}$, which takes the form 


$$
\left(\frac{\tau_{\max }}{\tau_{f}^{\prime}} \frac{\Delta \gamma}{2}+\frac{\sigma_{n, \max }}{\sigma_{f}^{\prime}} \frac{\Delta \varepsilon_{n}}{2}\right)_{\max }=\frac{\tau_{f}^{\prime}}{G}\left(\frac{2}{d_{f}}\right)^{2 b_{0}}+\gamma_{f}^{\prime}\left(\frac{2}{d_{f}}\right)^{2 c_{0}}
$$

where $\tau_{\max }$ and $\sigma_{n, \max }$ are the maximum shear stress and maximum normal stress, respectively. $\Delta \gamma / 2$ and $\Delta \varepsilon_{n} / 2$ are the shear strain amplitude and normal strain amplitude, respectively. $\tau_{f}^{\prime}$ and $\gamma_{f}^{\prime}$ are the shear fatigue strength and ductility constants, respectively. $\sigma_{f}^{\prime}$ is the fatigue strength constant. $G$ is the shear modulus. $b_{0}$ and $c_{0}$ are two exponents regarding fatigue strength and ductility, respectively. Note that the symbol ()$_{\max }$ on the left side of Eq. (18) represents the maximum fatigue damage factor for a determined critical plane.

\subsubsection{Creep damage based on modified SEDE}

In our previous works (Wang et al., 2017b; Wang et al., 2016; Wang et al., 2017c), the dominated parameters of failure strain energy density and creep strain energy density rate have been established to be adequate to ensure the accuracy of creep damage estimation. However, the framework of previous SEDE model is only applicable to uniaxial specimens. The original SEDE equation for creep damage in a specific cycle, $d_{c}$, is written as (Wang et al., 2017b)

$$
d_{c}=\int_{0}^{t_{h}}\left\{\frac{\dot{w}_{c}}{\min \left[w_{f}, w_{f, \text { trans }}(T)\right]}-\frac{\dot{w}_{c}}{w_{f, \text { trans }}(T)}\right\} d t
$$

where $t_{h}$ is the tensile hold time in one cycle, $\dot{w}_{c}$ is the creep strain energy density rate during a hold time period, $w_{f}$ is the failure strain energy density, and $w_{f, \text { trans }}(T)$ is the transition failure strain energy density under the condition free of creep damage at a certain temperature.

Two additional factors are needed to predict the creep damage under the multi- 
axial stress states in terms of the modified SEDE model. One is the elastic follow-up factor, $Z$, which is defined as a scalar to describe the magnitude of elastic follow-up phenomenon. Two special cases are generally found regarding the uniaxial creepfatigue experiment: (i) stress-controlled case during a hold time period causes a material to creep at a constant applied stress when $Z=\infty$, (ii) strain-controlled case during a hold time period causes a full stress relaxation at the same applied strain when $Z=1$, see Fig. 5. With respect to cases in between, the presence of elastic follow-up is a mechanical boundary condition lying between a stress controlled mode and a strain controlled mode (Wang et al., 2018b). The other factor is the multi-axial ductility factor, $M D F$, which provides a fundamental description for the effect of multi-axial stress states on the reduction of creep ductility (Wen et al., 2016).

The phenomenological Jeong's stress relaxation equation (Jeong et al., 2007) including the elastic follow-up factor is rewritten as

$$
\bar{\sigma}=\bar{\sigma}_{0}-\left(A \cdot \log \Delta \bar{\varepsilon}_{p p}+B\right) \cdot \log \left(1+\frac{t}{Z}\right)
$$

where $\bar{\sigma}_{0}$ is the equivalent peak tensile stress, $\Delta \bar{\varepsilon}_{p p}$ is the equivalent plastic strain range leading to pure fatigue, $\bar{\sigma}$ is the equivalent stress during a hold time period, $t$ is the time from start of hold time. $A$ and $B$ are two material-dependent constants. Compared with the strain-controlled mode $(Z=1)$ for uniaxial specimens, discontinuous geometric shapes of notched specimens are always subjected to mixed stress-strain controlled mode. In this case, the role of the elastic follow-up factor is to characterize the decrease of stress relaxation rate. The factor $Z$ is directly 
calculated by a segment length ratio of $\overline{\mathrm{KH}}$ and $\overline{\mathrm{KL}}$ within a relatively small hold time period, as seen in Fig. 5, which is also defined as a ratio of increased creep strain and released elastic strain during one hold time period. The instantaneous equivalent stress relaxation rate at a given time, $\dot{\bar{\sigma}}$, is given by the differentiation of Eq. (20), i.e.,

$$
\dot{\bar{\sigma}}=-\frac{A \cdot \log \Delta \bar{\varepsilon}_{p p}+B}{(Z+t) \cdot \ln 10}
$$

The area of stress-strain curve during a hold time period represents the drive force for creep damage based on the law of energy conservation (Fan et al., 2015), Fig. 5. In order to address the mean stress effect on creep damage estimation (Wang et al., 2017b; Wang et al., 2016; Wang et al., 2017c), the stress level higher than the negative magnitude of signed mean stress, i.e., $\sigma>-\bar{\sigma}_{m}$, during a hold time period is regarded as the driving force for creep damage, as schematically shown in Fig. 5. The highlighted area signifying the creep strain energy density, $w_{c}$, is expressed as

$$
w_{c}=\frac{Z}{2 \bar{E}} \cdot\left[\left(\bar{\sigma}_{0}\right)^{2}-(\bar{\sigma})^{2}+2 \bar{\sigma}_{m} \cdot \bar{\sigma}_{0}-2 \bar{\sigma}_{m} \cdot \bar{\sigma}\right]
$$

where $\bar{E}=3 E / 2(1+v)$. Then, instantaneous creep strain energy density rate at a given time, $\dot{w}_{c}$, can be obtained by differentiating Eq. (22) with respect to time, i.e.,

$$
\dot{w}_{c}=-\frac{Z \cdot \dot{\bar{\sigma}}}{\bar{E}} \cdot\left[\bar{\sigma}_{m}+\bar{\sigma}\right]
$$

Substituting Eq. (20) and Eq. (21) into Eq. (23) results in

$$
\dot{w}_{c}=\frac{Z \cdot M_{1}}{Z+t}-\frac{Z \cdot N_{1}}{Z+t} \cdot \log \left(1+\frac{t}{Z}\right)
$$

where 


$$
\begin{gathered}
M_{1}=\frac{\bar{\sigma}_{0} \cdot\left(A \cdot \log \Delta \bar{\varepsilon}_{p p}+B\right)}{\bar{E} \cdot \ln 10} \cdot \log \left(1+\frac{\bar{\sigma}_{m}}{\bar{\sigma}_{0}}\right) \\
N_{1}=\frac{\left(A \cdot \log \Delta \bar{\varepsilon}_{p p}+B\right)^{2}}{\bar{E} \cdot \ln 10}
\end{gathered}
$$

As mentioned before, $M D F$ is essential in creep damage estimation. A power law equation including the effect of multi-axial ductility, which is proposed to express the relationship between multi-axial failure strain energy density considering stress triaxiality, $w_{f}^{*}$, and instantaneous creep strain energy density rate, $\dot{w}_{c}$, can be expressed in the following two equations, i.e.,

$$
\begin{gathered}
w_{f}^{*}=w_{f} \cdot M D F \\
w_{f}=\varphi_{1}\left(\dot{w}_{c}\right)^{n_{1}}
\end{gathered}
$$

where $\varphi_{1}$ and $n_{1}$ are two material constants. It should be noted that the original definition of $M D F$ is given by the ratio of a multiaxial creep ductility to a uniaxial one, $\varepsilon_{f}^{*} / \varepsilon_{f}$. From Eq. (26a), MDF can also be defined as $w_{f}^{*} / w_{f}$, since the concept of strain energy density can be simply seen as the product of equivalent stress and creep ductility. In this case, failure strain energy density is proportional to creep ductility when stress state is fixed. Physically based Wen-Tu model (Wen et al., 2016) is introduced herein, where it is believed that the mechanism of creep damage is dominated by the initiation, growth and coalescence of micro-voids, i.e.,

$$
M D F=\exp \left[\frac{2}{3}\left(\frac{n_{2}-0.5}{n_{2}+0.5}\right)\right] / \exp \left[2\left(\frac{n_{2}-0.5}{n_{2}+0.5}\right) \frac{\sigma_{H}}{\bar{\sigma}}\right]
$$

where $n_{2}$ is the steady-state creep exponent and $\sigma_{H}$ is the hydrostatic stress. Replacing $w_{f}$ by $w_{f}^{*}$ and combing Eqs. (19), (24), (26a) and (26b), the creep damage per cycle can be rewritten as 


$$
d_{c}=\int_{0}^{t_{h}}\left\{\frac{\frac{Z \cdot M_{1}}{Z+t}-\frac{Z \cdot N_{1}}{Z+t} \cdot \log \left(1+\frac{t}{Z}\right)}{\min \left[\varphi_{1}\left[\frac{Z \cdot M_{1}}{Z+t}-\frac{Z \cdot N_{1}}{Z+t} \cdot \log \left(1+\frac{t}{Z}\right)\right]^{n_{1}} \cdot M D F, w_{f, \text { trans }}(T)\right]}-\frac{\frac{Z \cdot M_{1}}{Z+t}-\frac{Z \cdot N_{1}}{Z+t} \cdot \log \left(1+\frac{t}{Z}\right)}{w_{f, \text { trans }}(T)}\right\} d t
$$

\subsection{Finite element modelling for notched specimens}

A finite element modelling for the notched specimen is created using the commercial software ABAQUS so as to carry out the following cycle-by-cycle simulations. By virtue of symmetry and a reasonable computational consumption, only one quarter of the gauge-length area for each notch specimen was simulated in the FEA, as seen in Fig. 6. The meshing type was a 8-node linear brick (C3D8) element and the lateral length of each element at the notch root was approximately $0.3 \mathrm{~mm}$, matching up with the judgment criteria of the initial cracking (MeyerOlbersleben et al., 1999). Symmetry boundary conditions were employed on symmetry XOY and XOZ planes, respectively. Several nodes on the top line of XOY plane (namely the center line of the full FE modelling) were studied further since the notch side along the top line is regarded as the most dangerous region for the creepfatigue analysis. The global strain controlled mode was simulated in terms of a Zdirectional cyclic displacement load at the reference point which interacts with and consequently was constrained in the ending face of the finite element modelling.

\section{Results}

\subsection{Identification of the material parameters}

\subsubsection{Determination of the material parameters used in the NUVCM}


Within the initial elastic domain, material parameters $E, v$ and $Q_{0}$ can be easily obtained from the monotonic tensile curves for the GH4169 superalloy at $650{ }^{\circ} \mathrm{C}$. Three material parameters in the isotropic hardening rule, including $Q_{s a}, b$ and $H$, were determined following the procedure proposed by Saad et al (Saad et al., 2013). $K$ and $n$ in the viscous stress, $\sigma_{v}=K \dot{p}^{1 / n}$, were determined in terms of the monotonic tensile curves at different strain rates. The groups of $\left(\zeta_{i}, r_{i}\right)$ in the kinematic hardening rule were determined using the method suggested by Kang et al (Kang et al., 2002).

The rest material parameters in the modified static recovery term, especially the determination of $m(q)$ in Eq. (7) entails a detailed description. Firstly, the parameter $\gamma$ and loading-dependent parameter $m(q)$ under the creep-fatigue loading waveforms with various loading levels can be identified through the trial-and-error method. Then, with the aid of plastic strain memorization method, the magnitudes of plastic strain amplitude, $q$, were extracted to characterize the effect of loading level on stress relaxation behavior in the static recovery term. Finally, $\phi_{1}, \phi_{2}$ and $\omega$ of Eq. (7) can be determined in Fig. 7. It can be seen that the proposed exponential equation fit very well in different loading levels and the value of R-square is calculated to be 0.9985. In summary, all the material parameters used in the NUVCM involving elastic, viscoplastic, kinematic hardening and isotropic hardening have been shown in Table 3.

\subsubsection{Determination of the material parameters used in the damage models}

The current work regarding the extension of fatigue and creep damage models was 
based on the previous development, aiming at the creep-fatigue damage estimation for GH4169 at $650{ }^{\circ} \mathrm{C}$ (Wang et al., 2017b; Wang et al., 2019; Yu et al., 2017a). For all the material parameters used in the fatigue damage model based on CPM can be seen referred to (Yu et al., 2017a), where both the uniaxial and multi-axial fatigue tests have been validated, as summarized in Table 4. A series of creep and relaxation tests were used to fit the material constants in the creep damage model based on SEDE. For the detail regarding the fitting procedures as well as the validations of uniaxial creep-fatigue tests can be referred to (Wang et al., 2017b). The material constants for the estimation of creep damage are summarized in Table 4, where $n_{2}$ is used to calculate the value of $M D F$ and the rest of material constants have the same meanings as those in the previous SEDE models for uniaxial conditions.

\subsection{Validation of the NUVCM}

In this section, the NUVCM is validated in terms of hysteresis loops, stress relaxation curves during hold time periods and cyclic softening curves of specimens U-1 to U-4 with $\Delta \varepsilon_{t}$ ranging from $1.0 \%$ to $2.0 \%$ (Table 2). Fig. 8 shows the comparisons between the experimental data and simulation results of hysteresis loops at the $1^{\text {st }}$ cycle. The simulative shapes of hysteresis loops are predicted quite well with those of experimental ones. Specimen U-1 with the smallest $\Delta \varepsilon_{t}$ shows very limited plastic strain range, while the specimen U-4 with the largest $\Delta \varepsilon_{t}$ shows very significant plastic strain range, indicating that the selected loading levels of specimens U-1 to U-4 have covered almost the whole cyclic plastic behavior in this 
material. The comparisons between the experimental data and simulation results of stress relaxation curves at the $1^{\text {st }}$ cycle for specimens U-1 to U-4 are shown in Fig. 9. It can be seen that a good agreement between the experiments and the simulations has been achieved. Stress relaxation curves during hold time periods indicate that stress drops rapidly within the first several seconds and thereafter drops at a slow and steady rate. In addition, the absolute values of relaxed stress during the hold time periods decrease with decreasing loading levels, which can be well described by the fading exponent function in the static recovery term, $m(q)$, according to Eq. (7). The experimental data and the corresponding simulation results for cyclic softening curves are shown in Fig. 10. They are depicted in terms of the relationship between maximum stress for each cycle and the normalized life, $N / N_{f}$. The simulation results agree very well with the experimental cyclic softening data at an arbitrary total strain range, according to Eq. (11).

\subsection{Mesh independency analysis}

Mesh independency analysis is a necessary process to validate the feasibility of the proposed numerical procedure used in the notched specimens. The specimen $\mathrm{N}$ $10\left(\Delta \varepsilon_{t}=1.0 \%, t_{h}=60 \mathrm{~s}\right.$, Table 2$)$ is chosen as the typical specimen to analyze the mesh independency. From Fig. 6, we can see that the mesh size ranges from 0.3 to $1.0 \mathrm{~mm}$ from the notch root to the far end. Different meshing strategies and the corresponding damage results in the 1 st cycle are summarized in Table 5, in which the node located at the notch root is treated as the object of analysis. The name of 
"Global 1.0" represents that the meshing global size is $1.0 \mathrm{~mm}$ and no more mesh refinement is conducted in the current study case, while the name of "Gradient 0.3 to 1.0" denotes the meshing strategy used in Fig. 6. It can be seen from Table 5 that with refining the global mesh size, the magnitudes of fatigue damage, creep damage and total damage gradually increase. Another tendency is that when the global mesh size is refiner that $0.4 \mathrm{~mm}$ or "Gradient 0.3 to 1.0 ", the values of varies damages become steady, see Table 5. Considering the computational consumption and accuracy, the meshing strategy of "Gradient 0.3 to 1.0 " is the best solution among them.

\subsection{Experimental crack initiation life}

Both the uniaxial uniform and notched specimens under creep-fatigue loading waveforms for $\mathrm{GH} 4169$ at $650{ }^{\circ} \mathrm{C}$ are used to plot the crack initiation life distributions, as presented in Fig. 11. The data points of uniaxial uniform specimens with the hold times ranging from $0 \mathrm{~s}$ to $1800 \mathrm{~s}$ come from the previous work of (Wang et al., 2017b). As compared to uniaxial uniform specimens, creep-fatigue lives of notched specimens with the same loading level decreased to a greater extent. For notched specimens, more severe life reduction takes place with decreasing total strain range and increasing hold time, which can be regarded as a common trend of life distributions in various materials (Brinkman, 1985; Wang et al., 2018a). In particular, creep-fatigue endurances with a hold time of $3600 \mathrm{~s}$ reduce to approximately one tenth of the fatigue ones without hold periods. In comparison, 
uniaxial uniform specimens are impossible to cause such high life degradations. This indicates that stress concentration caused by geometric discontinuities can enhance the creep sensitivity to life reductions greatly.

\subsection{Metallographic observations of notched specimens}

Three notched specimens experiencing with different hold times at a given total strain range of $1.0 \%$ are chosen as typical examples to characterize crack initiation mechanisms, as shown in Fig. 12. From Figs. 12a and 12b, it can be clearly identified that crack initiates on the free surface of the notch root for specimen N-9 without a hold period ( $\Delta \varepsilon_{t}=1.0 \%, t_{h}=0 \mathrm{~s}$, Table 2) and specimen $\mathrm{N}-10$ with a short hold time $\left(\Delta \varepsilon_{t}=1.0 \%, t_{h}=60 \mathrm{~s}\right.$, Table 2). This is due to the fact that cyclic plastic deformation is higher at the surface with the role of stress concentration. On a micro level, the main driving force is attributed to the development of persistent slip bands near the surface due to a repetitive extrusion and intrusion process ( $\mathrm{Tu}$ and Zhang, 2016). Hence, specimens representing this type of loading condition and crack initiation mode are regarded as fatigue-damage-dominated specimens. On the contrary, the EBSD image of specimen N-12 with a long hold time $\left(\Delta \varepsilon_{t}=1.0 \%\right.$, $t_{h}=3600 \mathrm{~s}$, Table 2) provides the evidence for subsurface crack initiation sites, as depicted in Fig. 12c. In the early stage of crack initiation, isolated creep voids along grain boundaries and internal cracks at the tips of grain boundary wedge are captured far away from the surface (Telesman et al., 2016). At the same time, the slip band cracks at the notch surface seem to disappear. Thus, this kind of internal 
intergranular crack initiation mode is of typical creep damage, and specimen N-12 can be seen as a creep-damage-dominated specimen.

\section{Discussion}

\subsection{The stress-strain behavior of critical positions in the notched specimens}

The stress-strain analysis is focused on the vicinity of the notch root for three representative notched specimens, which is shown in Section 4.5 (specimen N-9, N$10, \mathrm{~N}-12$, Table 2) at a fixed total strain range of $1.0 \%$ and with different hold times (i.e., $0 \mathrm{~s}, 60 \mathrm{~s}$ and $3600 \mathrm{~s}$ ). The contours at the XOY plane of equivalent peak tensile stress $\bar{\sigma}_{0}$ and maximum principal strain $\varepsilon_{1}$ after a hold time period at the $100^{\text {th }}$ cycle are first shown in Fig. 13a and Fig. 13b, respectively. It can be seen that an increase in hold time almost has no influence on the magnitudes and maximum positions of $\bar{\sigma}_{0}$, while both the values and maximum positions of $\varepsilon_{1}$ change over hold time. As the hold times increase, it can be clearly observed that the maximum position of $\varepsilon_{1}$ gradually shifts from the notch root to an interior region. With this born in mind, the analysis of cyclic stress response and the accumulation of inelastic strain at the key points in the three representative notched specimens will be carried out in the following two subsections.

\subsubsection{Analysis of the cyclic stress response}

In order to further investigate the above-mentioned phenomenon, nodes 1 to 7 (Fig. 13) along the top line of the XOY plane (Fig. 6) for the three specimens 
(specimen $\mathrm{N}-9, \mathrm{~N}-10, \mathrm{~N}-12$, Table 2) are adopted to plot the relations between equivalent peak tensile stress in one cycle, $\bar{\sigma}_{0}$, and number of cycles, $N$, as seen in Figs. 14a-c. Very similar tendency and manifestation of $\bar{\sigma}_{0}$ for the same node in various specimens (without/with hold time periods) are shown in Figs. 14a-c, which indicate that the distribution and evolution of equivalent peak tensile stress in the notched specimens are independent of the lengths of hold time period. This phenomenon is consistent with equivalent peak tensile stress contours comparison in Fig. 13a-c. Another interesting phenomenon for each notched specimen is shown in Figs. 14a-c. Other than the continuous cyclic softening for uniform specimens (Fig. 10), the interior nodes near the notch root (i.e., nodes 3, 4, 5, Fig. 13) show an increased $\bar{\sigma}_{0}$ at the first several cycles. The gradient stress field results from the discontinuous geometric structure play an important role in this phenomenon. In addition, without considering the material behavior itself, the role of "residual stress field" in each cycle is to homogenize the gradient stress field, that is the high stress regions tend to decrease the current stress levels while the low stress regions have an opposite tendency (Chen et al., 2014; Giugliano et al., 2019; Gorash and Chen, 2013). Thus, very approaching magnitudes of $\bar{\sigma}_{0}$ for nodes 3,4 and 5 can be obtained at around $20^{\text {th }}$ cycle. After the period of structural cyclic stability, the material behavior of cyclic softening plays a dominant role in these curves.

\subsubsection{Cyclic accumulation of inelastic strain}

Similarly, the relations between maximum principal strain after a hold time period in each cycle, $\varepsilon_{1}$, and number of cycles, $N$, at the key nodes for the three specimens 
are respectively illustrated in Figs. 14d-f, indicating a gradual change in the maximum location of $\varepsilon_{1}$ with the increase of hold time. For all loading conditions, the highest values of $\varepsilon_{1}$ at the beginning several cycles occur at the notch root due to the presence of strain concentration. However, the introduction of hold time affects the growth rate of $\varepsilon_{1}$ per cycle, $\mathrm{d} \varepsilon_{1} / \mathrm{d} N$, for various nodes. For pure fatigue and creep-fatigue with very short hold times (specimen N-9 and N-10, Table 2), the similar magnitudes of $\mathrm{d} \varepsilon_{1} / \mathrm{d} N$ can be obtained among the nodes from 1 to 4 , and finally the highest value of $\varepsilon_{1}$ consistently locates at node 1 , as seen in Fig. $14 \mathrm{~d}$ and 14e. For creep-fatigue with long hold times (specimen N-12, Table 2), the highest $\mathrm{d} \varepsilon_{1} / \mathrm{d} N$ is located at node 3 . Up to the $100^{\text {th }}$ cycle, the value of $\varepsilon_{1}$ at node 3 overtakes that at node 1, as presented in Fig. 14f.

In order to investigate the fundamental reason for this tendency, the cycledependent hysteresis loops for the three specimens at nodes 1 and 3 are provided thereafter, while node 1 is at the notch root and node 3 may be at the most dangerous interior region, as plotted in Figs. 15a-c. Obvious cyclic accumulation of inelastic strain can only be observed for specimen N-10 and N-12, as shown in Figs. 15b and $15 \mathrm{c}$, respectively. Such a phenomenon may arise from many sources such as ratcheting, mode of control, creep and other known reasons (Taleb, 2013). Cycledependent hysteresis loops without a hold time for specimen N-9 (Fig. 15a) can be used to validate the contribution of ratcheting on the cyclic accumulation of inelastic strain. Ratcheting at a material level is traditionally defined as the cyclic accumulation of inelastic strain under a non-zero mean stress in each cycle 
(Chaboche, 2008; Kang, 2008). However, as shown in Fig. 15a, very small degree of negative directional ratcheting occurs due to the driving force of tiny negative mean stress for specimen N-9 (Abdel-Karim, 2009). Fig. 16 shows that tiny negative mean stress happens for specimen N-10 and N-12 with hold times of $60 \mathrm{~s}$ and $3600 \mathrm{~s}$, demonstrating that ratcheting also plays very limited negative role in the cyclic accumulation of tensile inelastic strain for the two creep-fatigue specimens. As compared to very clear positive directional cyclic accumulation of inelastic strain for the specimens under creep-fatigue conditions (Figs. 15b and 15c), the effect of traditional ratcheting can be virtually ignored.

For specimen $\mathrm{N}-10$ and $\mathrm{N}-12$, the stress-strain behavior during the hold time period at node 1 and 3 of the $100^{\text {th }}$ cycle are compared in the enlarged view of Fig. 15d. The slope of stress-strain curve during the hold time period represents the degree of controlling mode for each node. In detail, node 3 in the interior region appears to be more of a stress controlled mode (Fig. 5) than node 1 does at the notch root, tending to produce less stress relaxation rate as well as more obvious inelastic strain accumulation (Smith and Shirahatti, 2015; Wang et al., 2018b). From the viewpoint of a discontinuous geometric structure, it can also be characterized by elastic follow-up factor, $Z$, as shown in Fig. 5. It is due to the fact that the inhomogeneous stress fields around the notch root produces the misfit of creep/relaxation during hold time period (Ainsworth, 2003). In addition to this, the length of hold time is another factor dictating cyclic inelastic strain accumulation. If the hold time is no greater than $60 \mathrm{~s}$, like specimen N-10 in this work, the magnitude 
of equivalent inelastic strain range, $\Delta \bar{\varepsilon}_{i n}$, during the hold time period at node 3 is smaller than that at node 1 . This additional inelastic strain at node 3 is easier to recover in the reversed plastic period, leading to a limited value of $\mathrm{d} \varepsilon_{1} / \mathrm{d} N$. In this case, the initial value of $\varepsilon_{1}$ at the first cycle in fact determines that the position of the most serious cyclic accumulation of inelastic strain is located at node 1 , as shown in Fig. 14e. However, when the hold time is increased to $3600 \mathrm{~s}$, like specimen N-12, the higher value of $Z$ at node 3 plays a decisive role in a more significant inelastic strain accumulation. Thereafter, the highest value of $\mathrm{d} \varepsilon_{1} / \mathrm{d} N$ at node 3 in Fig. $14 \mathrm{f}$ can be well explained. The above-mentioned evolution process of the inelastic strain accumulation for different nodes in different specimens under various hold times is a strong evidence for the creep-cyclic plasticity and creep-fatigue interaction phenomenon.

\subsection{Life prediction for notched specimens}

After determining the stress-strain behavior of various notched specimens, the creep-fatigue interaction diagram is used for a further damage analysis. Three main aspects are included herein. Most importantly, the creep-fatigue interaction diagram on the basis of the proposed damage models is helpful in describing the cycledependent damage evolutions and in determining the crack initiation life afterwards. Secondly, the interaction diagram can well present the competitive relation between creep and fatigue under various loading conditions since it is composed of fatigue fraction and creep fraction in itself (Skelton and Gandy, 2014). Finally, the 
metallographic observations of location-dependent crack initiation sites in Section 4.5 are compared to the corresponding simulation results, depending on various loading conditions.

Figure 17a presents the cycle-dependent fatigue and creep damage evolutions in the creep-fatigue interaction diagram, where the accumulated fatigue damage $\sum_{i=1}^{j} d_{f}^{(i)}$ and the accumulated creep damage of $\sum_{i=1}^{j} d_{c}^{(i)}$ are respectively expressed as $D_{f}$ and $D_{c}$. Lines in different symbols denote the trajectories for cyclic damage evolutions under various creep-fatigue loading conditions. Once the trajectory for cyclic damage evolution intersects with the gray line representing the LDS rule, the crack initiation life can be determined. A general tendency in Fig. 17a visually shows that with increasing hold times and decreasing strain levels, creep damage becomes more dominant than others. Based on this observation, two types of creep-fatigue specimens, namely the fatigue-damage-dominated specimens and creep-damage-dominated specimens can be well distinguished. The crack initiation life prediction results for all the notched specimens subjected to fatigue and creep-fatigue loading conditions are shown in Fig. 17b. The predicted crack initiation results agree well with the experimental ones. All the data points lie within an error band of \pm 1.5 . With the aid of creep-fatigue interaction diagram, it can be clearly seen that if a trajectory closer to the vertical axis, creep damage will play a more dominant role in the process of crack initiation. 


\subsection{Simulated results of location-dependent crack initiation sites}

The competitive relation between creep and fatigue leading to the locationdependent crack initiation sites has been found in Section 4.5. The relations of fatigue/creep damage per cycle and normalized life for specimen $\mathrm{N}-10$ and specimen $\mathrm{N}-12$ at the notch root and the most dangerous interior node are presented in Fig. 18. Fatigue damage dominates the crack initiation process with a short hold time of $60 \mathrm{~s}$ for specimen N-10 (Fig. 18a), while creep damage takes over throughout the whole process with a long hold time of $3600 \mathrm{~s}$ for specimen N-12 (Fig. 18b). The corresponding damage contours on the XOZ plane (Fig. 6) show that the maximum accumulated fatigue damage is located at the notch root, i.e., node 1 while the max accumulated creep damage occurs at the interior region of node 3 . This tendency is consistent with the metallographic observations of EBSD maps around the notch root region, see Fig. 12.

Combined with the stress-strain behavior and the features of the current damage models, the simulation tendency in Fig. 18 can be explained as follows. Firstly, from the viewpoint of fatigue damage based on the CPM method, the critical plane at the notch root is always perpendicular to the free surface where the stress/strain tensors are not necessary to transform from global to local coordinate system by using the rotation matrixes (Chu, 1995). For the notched specimens in this work, it is also perpendicular to the loading direction, which means that the crack initiation behavior at the notch root is mainly controlled by the Z-directional stress/strain (Socie and Marquis, 2000). After that, considering the stress distribution gradient around the 
notch root, the maximum fatigue damage factor based on the left side of Eq. (18) reaches its extreme value on the free surface of the notch root. As a consequence, the maximum accumulated fatigue damage takes place at the notch root and the early crack under fatigue-damage-dominated conditions always initiates on the free surface of notch root.

Secondly, there are several reasons for the presence of the maximum accumulated creep damage at a somewhat interior region. In Fig. 19, the elastic follow-up factor and the $M D F$ are plotted against the normalized distance to notch root along the top line (Fig. 6) for specimen N-12 at the very last cycle before crack initiation. The attached black-to-white background is the contour for creep damage at the last cycle. The maximum value of $d_{c}$ near the notch root region is located at node 3 , experiencing the highest value of $Z$ and the lowest value of $M D F$. The elastic follow-up factor, which reflects the degree of mode of control (Fig. 5), has a great impact on creep damage. Firstly, the continuous cyclic accumulation of inelastic deformation at the interior node (node 3, Fig. 14f) inevitably leads to a tendency for crack initiation (Carroll et al., 2013). Secondly, if hold time period is closer to stress-controlled mode, the hysteresis area during a hold period representing driving force of creep damage (Fig. 5) will become larger since the relaxation rate is relatively slow. Last but not the least, stress triaxiality representing the effect of multi-axial stress states is remarkably higher at the interior region for notched specimens (Barbera et al., 2017), resulting in a lower magnitude of $M D F$, according to Eq. (27). In view of this, the interior region can exhibit a reduction in creep ductility and in failure strain energy density to a great 
extent (Wen et al., 2016).

Another feature of the history-dependent damage evolution is that the shifting of the most dangerous position with increasing number of cycles can be tracked. Take specimen $\mathrm{N}-12$ with a long hold time of $3600 \mathrm{~s}$ as an example, it can be clearly seen in Fig. 20 that the position of maximum accumulated total damage, $D_{f}+D_{c}$, is located at the notch root surface within the initial 20 cycles. However, this position shifts from the free surface to the interior region after the $22^{\text {th }}$ cycle due to the dominant role of creep damage under this loading condition (Fig. 18b). Compared to steady stressstrain analysis in the current assessments and design codes (Ainsworth, 2003; ASME, 2009; RCC-MRx, 2012), this history-dependent damage evolution technique is of great significance to meet the requirement of irregular loading waveforms, overloading conditions as well as the remaining lifetime assessment in practice. Two main aspects based on this paper will be investigated in our further work. On is that the accelerated algorithm must be implemented into the current numerical procedure due to a high computational expense for each study case. The other is that although the final location-dependent crack initiation sites under various loading conditions can be well identified, the shifting of the most dangerous position before crack initiation for a specified specimen warrants a follow-up study via the in-situ creep-fatigue testing technique.

\section{Conclusions}

Strain-controlled cyclic tests were carried out on uniform and notched specimens 
for the nickel-based GH4169 superalloy at $650{ }^{\circ} \mathrm{C}$. The proposed numerical procedure involving modified unified viscoplastic constitutive model and damage models is presented for the creep-fatigue analysis under multi-axial stress states. The main results and finding are listed as follows:

(1) A new unified viscoplastic constitutive model involving a modified Chaboche's nonlinear kinematic hardening rule and an isotropic hardening rule is developed to describe the cyclic softening and stress relaxation behavior. The predicted cyclic softening curves, stress relaxation curves and hysteresis stress-strain loops in the uniaxial specimens are in good agreement with those of experiments.

(2) A critical plane-based fatigue damage parameter is presented for fatigue damage. A strain energy density exhaustion model considering both an elastic follow-up factor and a multi-axial ductility factor is proposed for creep damage. With the validation of notched specimens, the proposed models show a reasonable crack initiation prediction results within an error band of \pm 1.5 .

(3) The stress-strain behavior around the notch root region shows a mixed controlled mode under global strain-controlled creep-fatigue loading conditions. In contract to pure fatigue tests without hold time periods, obvious cyclic accumulation of inelastic strain caused by the inhomogeneous stress fields can be obtained for creep-fatigue tests with long hold times.

(4) Location-dependent crack initiation sites are revealed using the proposed numerical procedure, which is in consistency with the corresponding metallographic observations via the electron backscatter diffraction technique. 
Crack initiates from the surface of notch for each fatigue-damage-dominated notched specimen, while it initiates from the subsurface for each creep-damagedominated notched specimen. The degree of controlling mode and stress triaxiality are responsible for this phenomenon. 


\section{Acknowledgements}

The authors would like to acknowledge gratefully for the financial support through National Natural Science Foundations of China (51725503, 11872181, 51828501) and the 111 project. The author X.C. Zhang is also grateful for the support by Shanghai Technology Innovation Program of SHEITC (CXY-2015-001), Fok Ying Tung Education Foundation, and Young Program of Yangtze River Scholars. The author S.J. Guo is also grateful for the Shanghai Natural Science Foundation (18ZR1408900). 


\section{References}

Abdel-Karim, M., 2009. Modified kinematic hardening rules for simulations of ratchetting. International Journal of Plasticity 25, 1560-1587.

Ahmed, R., Barrett, P.R., Hassan, T., 2016. Unified viscoplasticity modeling for isothermal low-cycle fatigue and fatigue-creep stress-strain responses of Haynes 230. International Journal of Solids and Structures 88-89, 131-145.

Ainsworth, R., 2003. R5: Assessment procedure for the high temperature response of structures. British Energy Generation Ltd 3.

ASME, 2009. ASME Boiler and Pressure Vessel Code, Section III, Division 1, Subsection NH, Class 1 Components in Elevated Temperature Service. American Society of Mechanical Engineers, New York, NY.

Barbera, D., Chen, H., Liu, Y., 2017. Advances on creep-fatigue damage assessment in notched components. Fatigue \& Fracture of Engineering Materials \& Structures 40, 1854-1867.

Barrett, R.A., Farragher, T.P., Hyde, C.J., O'Dowd, N.P., O'Donoghue, P.E., Leen, S.B., 2014a. A Unified Viscoplastic Model for High Temperature Low Cycle Fatigue of Service-Aged P91 Steel. Journal of Pressure Vessel Technology 136, 021402.

Barrett, R.A., Farragher, T.P., O’Dowd, N.P., O’Donoghue, P.E., Leen, S.B., 2013 a. Multiaxial cyclic viscoplasticity model for high temperature fatigue of P91 steel. Materials Science and Technology 30, 67-74.

Barrett, R.A., O'Donoghue, P.E., Leen, S.B., 2017. A physically-based constitutive 
model for high temperature microstructural degradation under cyclic deformation. International Journal of Fatigue 100, 388-406.

Barrett, R.A., O’Donoghue, P.E., Leen, S.B., 2013b. An improved unified viscoplastic constitutive model for strain-rate sensitivity in high temperature fatigue. International Journal of Fatigue 48, 192-204.

Barrett, R.A., O’Donoghue, P.E., Leen, S.B., 2014b. A dislocation-based model for high temperature cyclic viscoplasticity of $9-12 \mathrm{Cr}$ steels. Computational Materials Science 92, 286-297.

Bonnand, V., Chaboche, J., Cherouali, H., Kanoute, P., Ostoja-Kuczynski, E., Vogel, F., 2010. Investigation of multiaxial fatigue in the prospect of turbine disc applications: Part II-Fatigue criteria analysis and formulation of a new combined one, ICMFF9.

Brinkman, C.R., 1985. High-temperature time-dependent fatigue behaviour of several engineering structural alloys. International metals reviews 30, 235-258.

Carroll, L.J., Cabet, C., Carroll, M.C., Wright, R.N., 2013. The development of microstructural damage during high temperature creep-fatigue of a nickel alloy. International Journal of Fatigue 47, 115-125.

Chaboche, J., Van, K.D., Cordier, G., 1979. Modelization of the strain memory effect on the cyclic hardening of 316 stainless steel.

Chaboche, J.L., 2008. A review of some plasticity and viscoplasticity constitutive theories. International Journal of Plasticity 24, 1642-1693.

Chen, H., Chen, W., Ure, J., 2014. A direct method on the evaluation of cyclic 
steady state of structures with creep effect. Journal of Pressure Vessel Technology 136, 061404.

Chen, L., Jiang, J., Fan, Z., Chen, X., Yang, T., 2007. A new model for life prediction of fatigue-creep interaction. International Journal of Fatigue 29, 615619.

Chen, W., Feng, M., 2015. A study of a cyclic viscoplasticity model based on hyperbolic sine form for the inelastic strain rate. International Journal of Mechanical Sciences 101-102, 155-160.

Chen, W., Wang, F., Feng, M., 2017. Study of a modified non-unified model for time-dependent behavior of metal materials. Mechanics of Materials 113, 69-76.

Chu, C.-C., 1995. Fatigue damage calculation using the critical plane approach. Journal of Engineering Materials and Technology 117, 41-49.

Dunne, F.P.E., 2014. Fatigue crack nucleation: Mechanistic modelling across the length scales. Current Opinion in Solid State and Materials Science 18, 170-179.

Fan, Y.-N., Shi, H.-J., Tokuda, K., 2015. A generalized hysteresis energy method for fatigue and creep-fatigue life prediction of $316 \mathrm{~L}(\mathrm{~N})$. Materials Science and Engineering: A 625, 205-212.

Fatemi, A., Socie, D.F., 1988. A critical plane approach to multiaxial fatigue damage including out - of - phase loading. Fatigue \& Fracture of Engineering Materials \& Structures 11, 149-165.

Findley, W.N., 1958. A theory for the effect of mean stress on fatigue of metals under combined torsion and axial load or bending. Engineering Materials 
Research Laboratory, Division of Engineering, Brown University.

Giugliano, D., Barbera, D., Chen, H., Cho, N.-K., Liu, Y., 2019. Creep-fatigue and cyclically enhanced creep mechanisms in aluminium based metal matrix composites. European Journal of Mechanics-A/Solids 74, 66-80.

Gorash, Y., Chen, H., 2013. A parametric study on creep-fatigue strength of welded joints using the linear matching method. International Journal of Fatigue 55, $112-125$.

Hales, R., 1980. A quantitative metallographic assessment of structural degradation of type 316 stainless steel during creep-fatigue. Fatigue \& Fracture of Engineering Materials \& Structures 3, 339-356.

Ince, A., Glinka, G., 2014. A generalized fatigue damage parameter for multiaxial fatigue life prediction under proportional and non-proportional loadings. International Journal of Fatigue 62, 34-41.

Jeong, C.Y., Bae, J.-C., Kang, C.-S., Cho, J.-I., Son, H.-T., 2007. Normalized creepfatigue life prediction model based on the energy dissipation during hold time. Materials Science and Engineering: A 460-461, 195-203.

Jordon, J.B., Bernard, J.D., Newman, J.C., 2012. Quantifying microstructurally small fatigue crack growth in an aluminum alloy using a silicon-rubber replica method. International Journal of Fatigue 36, 206-210.

Kang, G., 2008. Ratchetting: Recent progresses in phenomenon observation, constitutive modeling and application. International Journal of Fatigue 30, 14481472. 
Kang, G., Gao, Q., Yang, X., 2002. A visco-plastic constitutive model incorporated with cyclic hardening for uniaxial/multiaxial ratcheting of SS304 stainless steel at room temperature. Mechanics of Materials 34, 521-531.

Kang, G., Kan, Q., 2007. Constitutive modeling for uniaxial time-dependent ratcheting of SS304 stainless steel. Mechanics of Materials 39, 488-499.

Kobayashi, M., Mukai, M., Takahashi, H., Ohno, N., Kawakami, T., Ishikawa, T., 2003. Implicit integration and consistent tangent modulus of a time - dependent non - unified constitutive model. International Journal for Numerical Methods in Engineering 58, 1523-1543.

Lemaitre, J., Chaboche, J.-L., 1990. Mechanics of solid materials. Cambridge university press.

Manson, S., 1968. A simple procedure for estimating high-temperature low-cycle fatigue. Experimental Mechanics 8, 349-355.

Meyer-Olbersleben, F., Kasik, N., Ilschner, B., Rezai-Aria, F., 1999. The thermal fatigue behavior of the combustor alloys IN 617 and Haynes 230 before and after welding. Metallurgical and Materials Transactions A 30, 981-989.

Naumenko, K., Altenbach, H., 2007. Modeling of creep for structural analysis. Springer Science \& Business Media.

Nouailhas, D., 1989. Unified modelling of cyclic viscoplasticity: application to austenitic stainless steels. International journal of plasticity 5, 501-520.

Pineau, A., McDowell, D.L., Busso, E.P., Antolovich, S.D., 2016. Failure of metals II: Fatigue. Acta Materialia 107, 484-507. 
Priest, R.H., Ellison, E.G., 1981. A combined deformation map-ductility exhaustion approach to creep-fatigue analysis. Materials Science and Engineering 49, 7-17. RCC-MRx, 2012. Construction Rules for mechanical components of nuclear installations. AFCEN: Paris, France.

Robinson, E.L., 1952. Effect of temperature variation on the long-time rupture strength of steels. Trans. ASME 74, 777-781.

Saad, A.A., Hyde, T., Sun, W., Hyde, C.J., Tanner, D.W., 2013. Characterization of viscoplasticity behaviour of P91 and P92 power plant steels. International Journal of Pressure Vessels and Piping 111, 246-252.

Sangid, M.D., 2013. The physics of fatigue crack initiation. International Journal of Fatigue 57, 58-72.

Skelton, R.P., Gandy, D., 2014. Creep - fatigue damage accumulation and interaction diagram based on metallographic interpretation of mechanisms. Materials at High Temperatures 25, 27-54.

Smith, D.J., Shirahatti, A.M., 2015. The effects of long-range residual stress, elastic follow-up and applied load on creep crack incubation and material toughness. The Journal of Strain Analysis for Engineering Design 50, 455-469.

Smith, K.N., 1970. A stress-strain function for the fatigue of metals. Journal of materials 5, 767-778.

Socie, D.F., Marquis, G.B., 2000. Multiaxial fatigue. Society of Automotive Engineers Warrendale, PA.

Spindler, M.W., 2005. The prediction of creep damage in type 347 weld metal. Part I: 
the determination of material properties from creep and tensile tests. International Journal of Pressure Vessels and Piping 82, 175-184.

Spindler, M.W., Payten, W.M., 2011. Advanced ductility exhaustion methods for the calculation of creep damage during creep-fatigue cycling. Journal of ASTM International 8, 1-19.

Susmel, L., 2010. A simple and efficient numerical algorithm to determine the orientation of the critical plane in multiaxial fatigue problems. International Journal of Fatigue 32, 1875-1883.

Takahashi, Y., Dogan, B., Gandy, D., 2009. Systematic evaluation of creep-fatigue life prediction methods for various alloys, ASME 2009 Pressure Vessels and Piping Conference. American Society of Mechanical Engineers, pp. 1461-1470.

Taleb, L., 2013. About the cyclic accumulation of the inelastic strain observed in metals subjected to cyclic stress control. International Journal of Plasticity 43, 119.

Telesman, J., Gabb, T.P., Ghosn, L.J., Gayda, J., 2016. Effect of notches on creepfatigue behavior of a $\mathrm{P} / \mathrm{M}$ nickel-based superalloy. International Journal of Fatigue 87, 311-325.

Tu, S., Zhang, X., 2016. Fatigue Crack Initiation Mechanisms. Reference Module in Materials Science and Materials Engineering, 1-23.

Wang, C., Brown, M., 1993. A path - independent parameter for fatigue under proportional and non - proportional loading. Fatigue \& fracture of engineering materials \& structures $16,1285-1297$. 
Wang, C., Brown, M., 1996. Life prediction techniques for variable amplitude multiaxial fatigue-part 1: theories. Journal of Engineering Materials and Technology 118, 367-370.

Wang, C., Shi, D., Yang, X., Li, S., Dong, C., 2017a. An improved viscoplastic constitutive model and its application to creep behavior of turbine blade. Materials Science and Engineering: A 707, 344-355.

Wang, P., Cui, L., Lyschik, M., Scholz, A., Berger, C., Oechsner, M., 2012. A local extrapolation based calculation reduction method for the application of constitutive material models for creep fatigue assessment. International Journal of Fatigue 44, 253-259.

Wang, R.-Z., Wang, J., Gong, J.-G., Zhang, X.-C., Tu, S.-T., Zhang, C.-C., 2018a. Creep-Fatigue Behaviors and Life Assessments in Two Nickel-Based Superalloys. Journal of Pressure Vessel Technology 140, 031405.

Wang, R.-Z., Zhang, X.-C., Gong, J.-G., Zhu, X.-M., Tu, S.-T., Zhang, C.-C., 2017b. Creep-fatigue life prediction and interaction diagram in nickel-based GH4169 superalloy at $650^{\circ} \mathrm{C}$ based on cycle-by-cycle concept. International Journal of Fatigue 97, 114-123.

Wang, R.-Z., Zhang, X.-C., Tu, S.-T., Zhu, S.-P., Zhang, C.-C., 2016. A modified strain energy density exhaustion model for creep-fatigue life prediction. International Journal of Fatigue 90, 12-22.

Wang, R.-Z., Zhu, S.-P., Wang, J., Zhang, X.-C., Tu, S.-T., Zhang, C.-C., 2019. High temperature fatigue and creep-fatigue behaviors in a Ni-based superalloy: 
Damage mechanisms and life assessment. International Journal of Fatigue 118, 8-21.

Wang, R.-Z., Zhu, X.-M., Zhang, X.-C., Tu, S.-T., Gong, J.-G., Zhang, C.-C., 2017c. A generalized strain energy density exhaustion model allowing for compressive hold effect. International Journal of Fatigue 104, 61-71.

Wang, Y.Q., Coules, H.E., Truman, C.E., Smith, D.J., 2018b. Effect of elastic follow-up and ageing on the creep of an austenitic stainless steel. International Journal of Solids and Structures 135, 219-232.

Wen, J.-F., Srivastava, A., Benzerga, A., Tu, S.-T., Needleman, A., 2017. Creep crack growth by grain boundary cavitation under monotonic and cyclic loading. Journal of the Mechanics and Physics of Solids 108, 68-84.

Wen, J.-F., Tu, S.-T., Xuan, F.-Z., Zhang, X.-W., Gao, X.-L., 2016. Effects of Stress Level and Stress State on Creep Ductility: Evaluation of Different Models. Journal of Materials Science \& Technology 32, 695-704.

Yaguchi, M., Yamamoto, M., Ogata, T., 2002. A viscoplastic constitutive model for nickel-base superalloy, part 1: kinematic hardening rule of anisotropic dynamic recovery. International Journal of Plasticity 18, 1083-1109.

Yang, Q.D., Schesser, D., Niess, M., Wright, P., Mavrogordato, M.N., Sinclair, I., Spearing, S.M., Cox, B.N., 2015. On crack initiation in notched, cross-plied polymer matrix composites. Journal of the Mechanics and Physics of Solids 78, 314-332.

Yu, Z.Y., Zhu, S.P., Liu, Q., Liu, Y., 2017a. Multiaxial Fatigue Damage Parameter 
and Life Prediction without Any Additional Material Constants. Materials 10, 923-938.

Yu, Z.Y., Zhu, S.P., Liu, Q., Liu, Y., 2017b. A New Energy-Critical Plane Damage Parameter for Multiaxial Fatigue Life Prediction of Turbine Blades. Materials $10,513-531$.

Zhang, S.-L., Xuan, F.-Z., 2017. Interaction of cyclic softening and stress relaxation of $9-12 \%$ Cr steel under strain-controlled fatigue-creep condition: Experimental and modeling. International Journal of Plasticity 98, 45-64. 


\section{List of Figure captions}

Figure 1 Preparation processes of uniaxial and notched specimens under cyclic conditions.

Figure 2 Shapes and dimensions of (a) uniaxial specimens and (b) notched specimens used in the cyclic tests.

Figure 3 Global strain-controlled loading condition for notched specimens on the MTS testing machine.

Figure 4 Overall flow chart of the crack initiation life assessment for notched specimens.

Figure 5 Schematic diagram illustrating mixed stress-strain controlled mode, definition of elastic follow-up factor and driving force for creep damage.

Figure 6 FE model, meshing and boundary conditions of the notched specimens.

Figure 7 Fitting the relation between the exponent controlling the static recovery and plastic strain amplitude.

Figure 8 Experimental data and simulation results of hysteresis loops at the $1^{\text {st }}$ cycle for specimens $\mathrm{U}-1$ to $\mathrm{U}-4$.

Figure 9 Experimental data and simulation results of stress relaxation curves at the $1^{\text {st }}$ cycle for specimens U-1 to U-4.

Figure 10 Experimental data and simulation results of cyclic softening curves for specimens $\mathrm{U}-1$ to $\mathrm{U}-4$. 
Figure 11 Experimental crack initiation life distributions of uniaixal uniform and notched specimens under creep-fatigue loading waveforms for GH4169 at $650{ }^{\circ} \mathrm{C}$.

Figure 12 EBSD maps of main crack initiation for (a) specimen N-10 $\left(\Delta \varepsilon_{t}=1.0 \%\right.$, $\left.t_{h}=60 \mathrm{~s}\right)$ and $(\mathrm{b})$ specimen $\mathrm{N}-12\left(\Delta \varepsilon_{t}=1.0 \%, t_{h}=3600 \mathrm{~s}\right)$.

Figure 13 The contours at XOY plane of (a) equivalent peak tensile stress, $\bar{\sigma}_{0}$, and (b) maximum principal strain after hold time period, $\varepsilon_{1}$ at the $100^{\text {th }}$ cycle for specimen $\mathrm{N}-9\left(\Delta \varepsilon_{t}=1.0 \%, t_{h}=0 \mathrm{~s}\right)$, specimen $\mathrm{N}-10\left(\Delta \varepsilon_{t}=1.0 \%\right.$, $\left.t_{h}=60 \mathrm{~s}\right)$ and specimen N-12 $\left(\Delta \varepsilon_{t}=1.0 \%, t_{h}=3600 \mathrm{~s}\right)$.

Figure 14 (a) The relations between equivalent tensile peak stress and number of cycles at various nodes for (a) specimen N-9 $\left(\Delta \varepsilon_{t}=1.0 \%, t_{h}=0 \mathrm{~s}\right)$, (b) specimen $\mathrm{N}-10\left(\Delta \varepsilon_{t}=1.0 \%, t_{h}=60 \mathrm{~s}\right)$, and (c) specimen $\mathrm{N}-12$ $\left(\Delta \varepsilon_{t}=1.0 \%, t_{h}=3600 \mathrm{~s}\right)$; and the relations between maximum principal strain and number of cycles for (d) specimen N-9, (e) specimen N-10, and (f) specimen $\mathrm{N}-12$.

Figure 15 Vertical panel figures of cycle-dependent hysteresis loops at nodes 1 and 3 for (a) specimen N-9 $\left(\Delta \varepsilon_{t}=1.0 \%, t_{h}=0 \mathrm{~s}\right)$, (b) specimen $\mathrm{N}-10$ $\left(\Delta \varepsilon_{t}=1.0 \%, t_{h}=60 \mathrm{~s}\right)(\mathrm{c})$ specimen $\mathrm{N}-12\left(\Delta \varepsilon_{t}=1.0 \%, t_{h}=3600 \mathrm{~s}\right)$, and (d) the enlarged view of stress-strain behavior during hold time period at node 1 and 3 of the $100^{\text {th }}$ cycle for specimen $\mathrm{N}-10$ and specimen $\mathrm{N}-12$. 
Figure 16 The evolution of mean stress with various numbers of cycles at node 1 of notch root for specimen $\mathrm{N}-9\left(\Delta \varepsilon_{t}=1.0 \%, t_{h}=0 \mathrm{~s}\right)$, specimen $\mathrm{N}-10$ $\left(\Delta \varepsilon_{t}=1.0 \%, t_{h}=60 \mathrm{~s}\right)$, and specimen N-12 $\left(\Delta \varepsilon_{t}=1.0 \%, t_{h}=3600 \mathrm{~s}\right)$.

Figure 17 (a) The trajectories for cyclic damage evolutions in the creep-fatigue interaction diagram, and (b) the prediction capacities by using the damage models.

Figure 18 The relations of fatigue/creep damage per cycle and normalized life and corresponding accumulated damage contours at XOZ plane at the last cycle before crack initiation for (a) specimen N-10 $\left(\Delta \varepsilon_{t}=1.0 \%, t_{h}=60 \mathrm{~s}\right)$ and (b) specimen N-12 $\left(\Delta \varepsilon_{t}=1.0 \%, t_{h}=3600 \mathrm{~s}\right)$.

Figure 19 The relations of elastic follow-up factor and the $M D F$ against normalized distance away from the notch root along the top line for specimen $\mathrm{N}-12$ $\left(\Delta \varepsilon_{t}=1.0 \%, t_{h}=3600 \mathrm{~s}\right)$.

Figure 20 The shifting of most dangerous position with increasing number of cycles for specimen $\mathrm{N}-12\left(\Delta \varepsilon_{t}=1.0 \%, t_{h}=3600 \mathrm{~s}\right)$. 


\section{List of Table captions}

Table 1 Chemical compositions (wt.\%) of the nickel-based GH4169 superalloy

Table 2 Summary of input parameters for uniaxial and notched specimens in cyclic tests.

Table 3 Material constants used in the unified viscoplastic constitutive model for GH4169 superalloy at $650{ }^{\circ} \mathrm{C}$.

Table 4 Material constants used in the fatigue damage model based on CPM and creep damage based on modified SEDE for GH4169 superalloy at $650{ }^{\circ} \mathrm{C}$.

Table 5 Mesh independency analysis for specimen N-10 $\left(\Delta \varepsilon_{t}=1.0 \%, t_{h}=60 \mathrm{~s}\right.$, Table 2). 


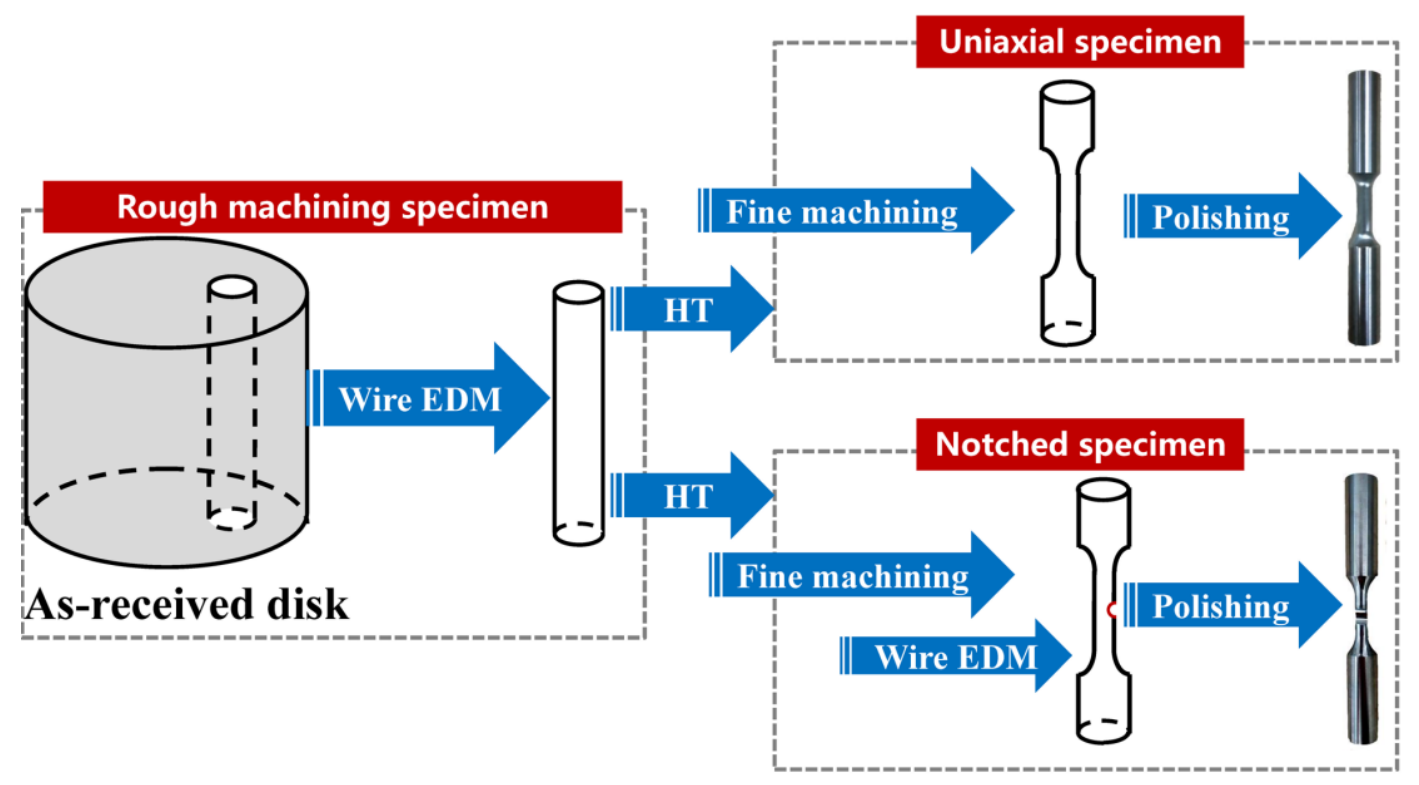

Figure 1 Preparation processes of uniaxial and notched specimens in cyclic tests. 
(a)

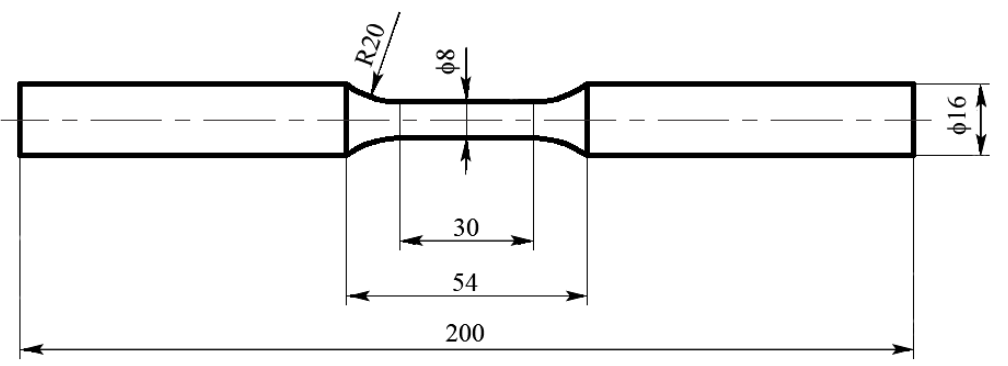

Unit: mm

(b)

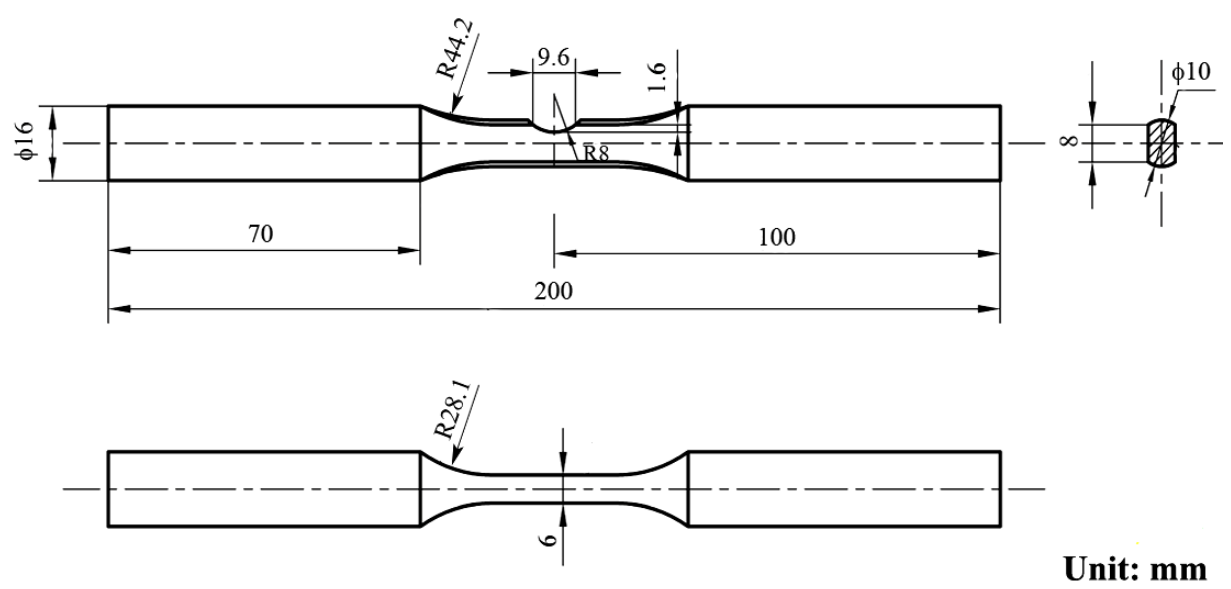

Figure 2 Shapes and dimensions of (a) uniaxial specimens and (b) notched specimens used in the cyclic tests. 


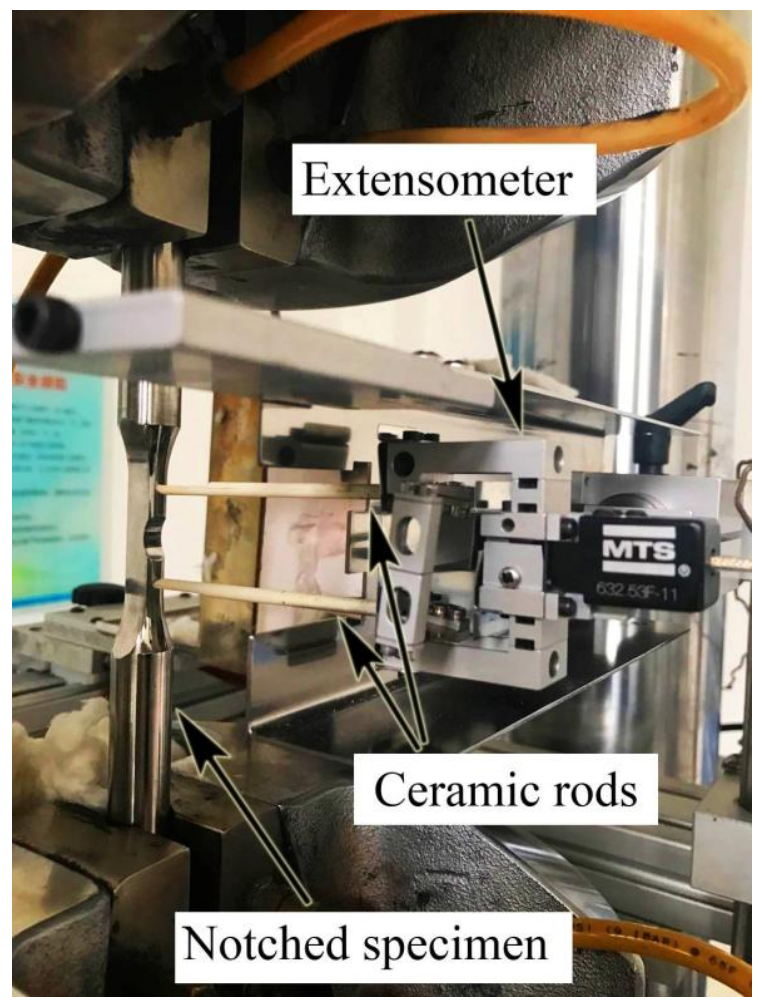

Figure 3 Global strain-controlled loading condition for notched specimens on the MTS testing machine. 


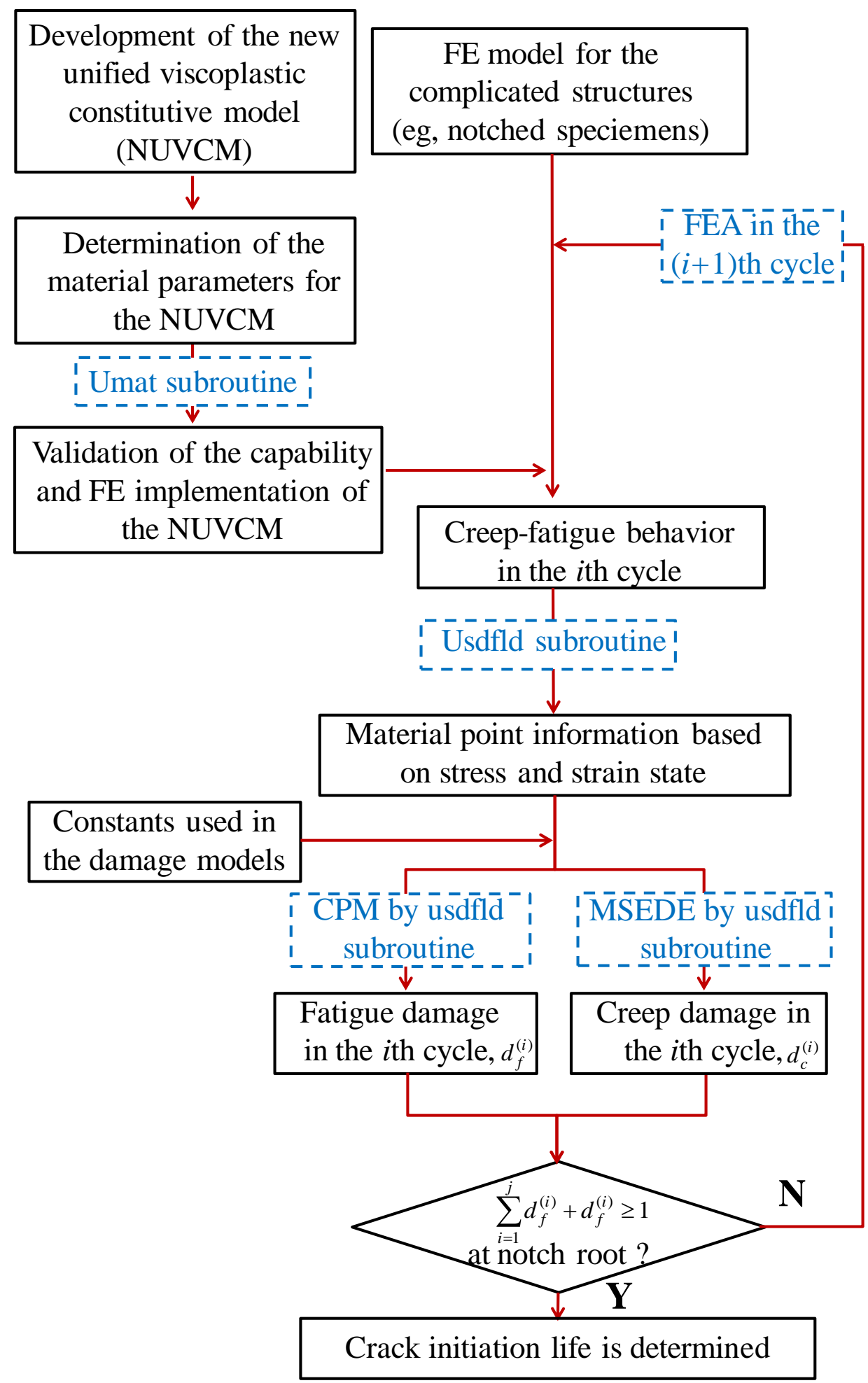

Figure 4 Overall flow chart of the crack initiation life assessment for notched specimens. 


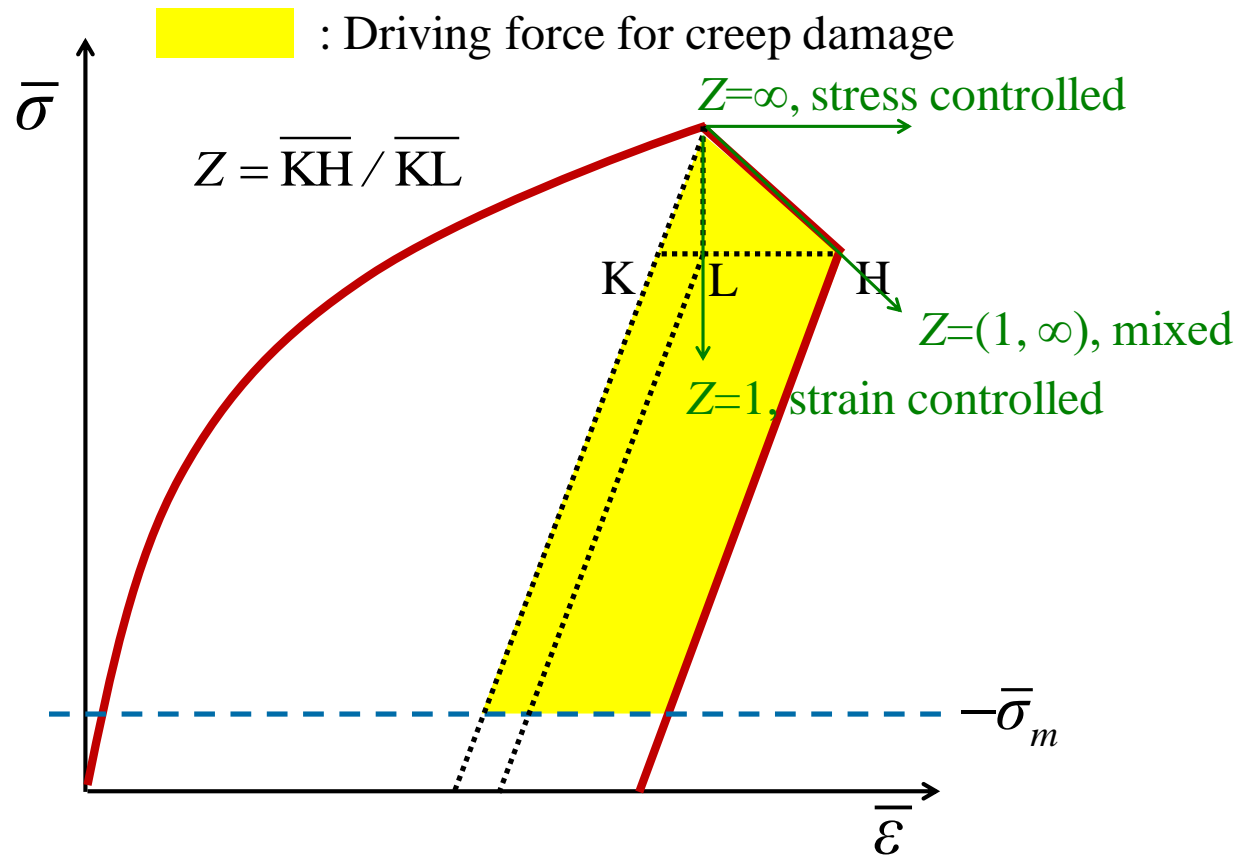

Figure 5 Schematic diagram illustrating mixed stress-strain controlled mode, definition of elastic follow-up factor and driving force for creep damage. 


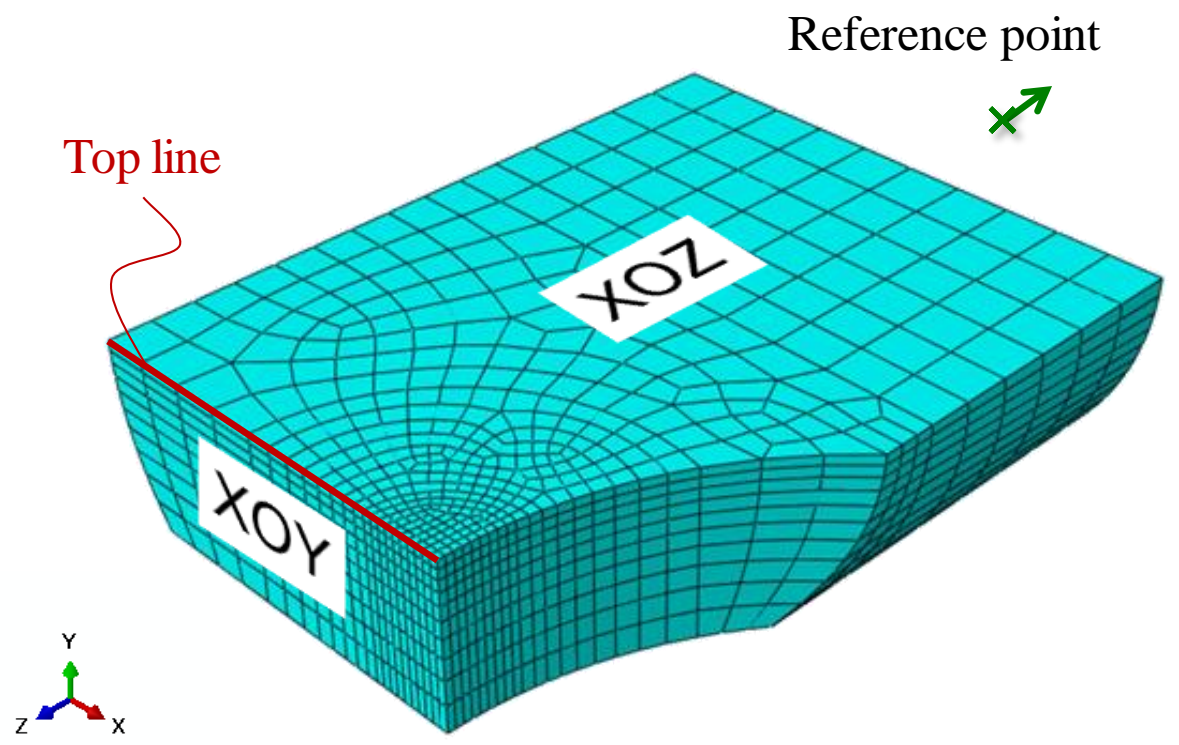

Figure $6 \mathrm{FE}$ model, meshing and boundary conditions of the notched specimens 


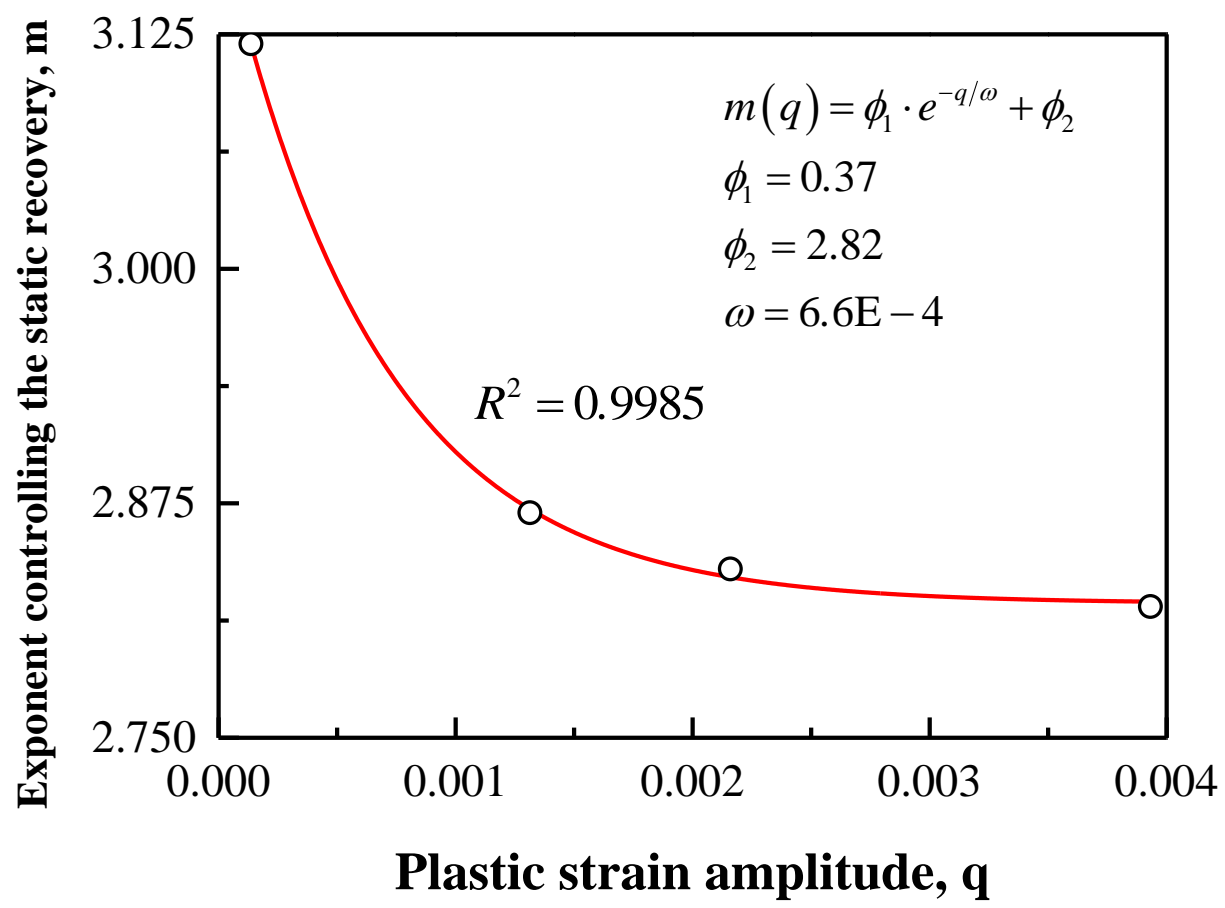

Figure 7 Fitting the relation between the exponent controlling the static recovery and plastic strain amplitude. 


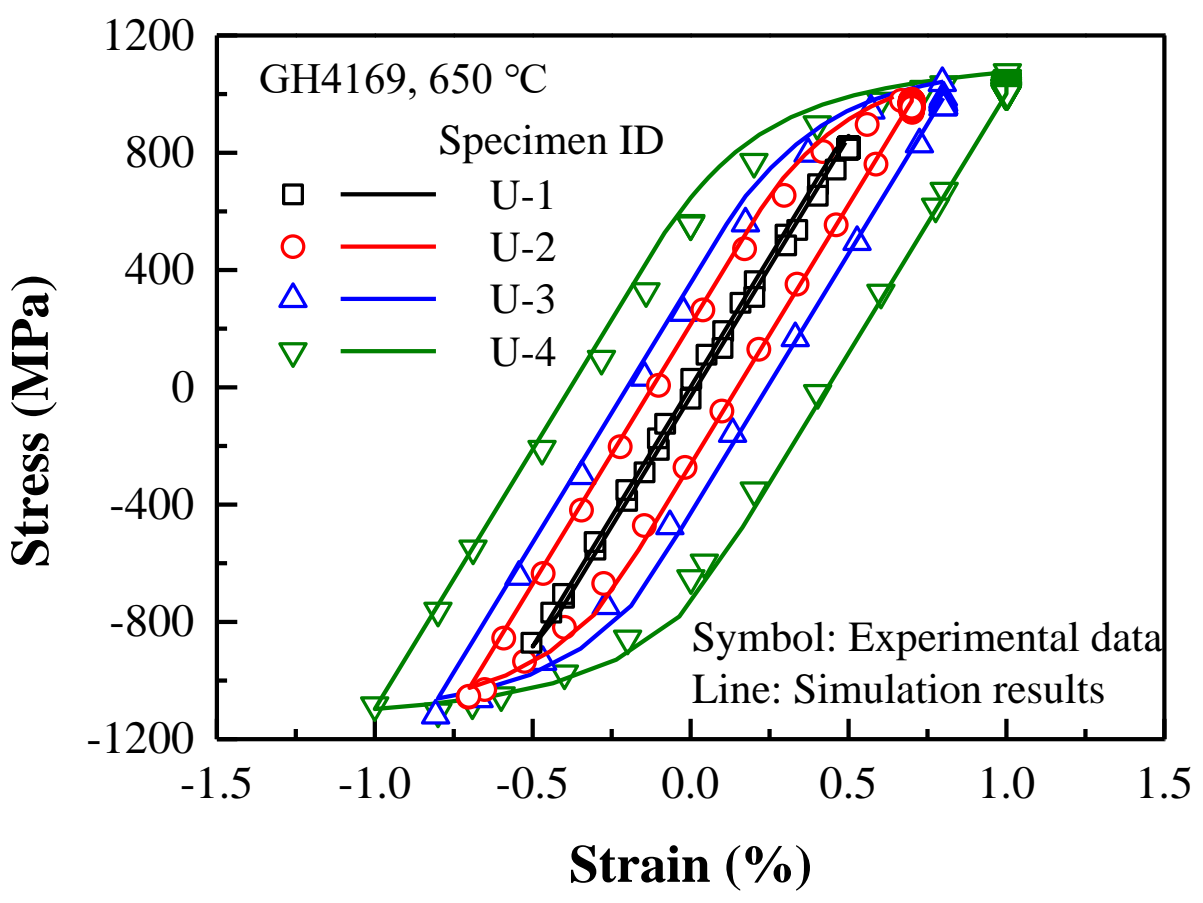

Figure 8 Experimental data and simulation results of hysteresis loops at the $1^{\text {st }}$ cycle for specimens $\mathrm{U}-1$ to $\mathrm{U}-4$. 


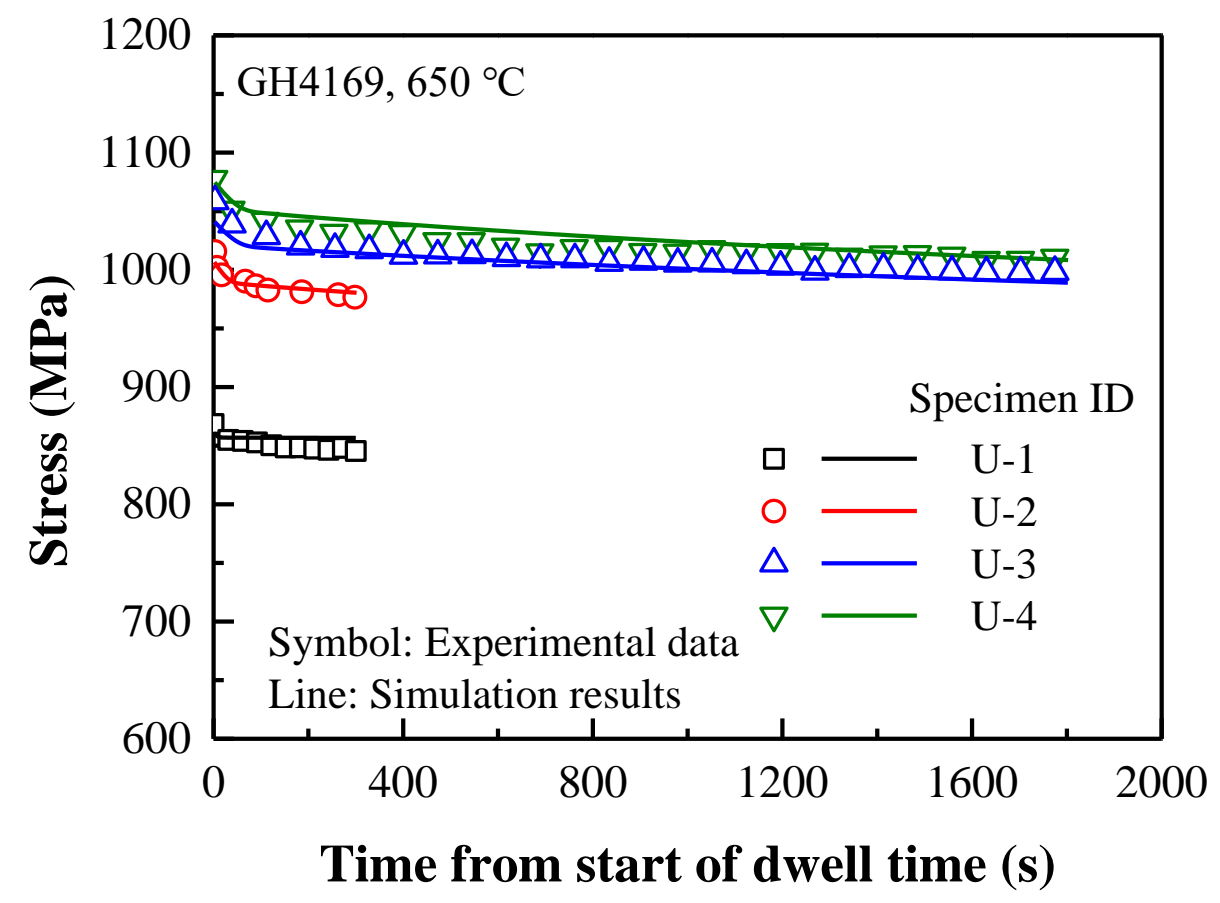

Figure 9 Experimental data and simulation results of stress relaxation curves at the $1^{\text {st }}$ cycle for specimens U-1 to U-4. 


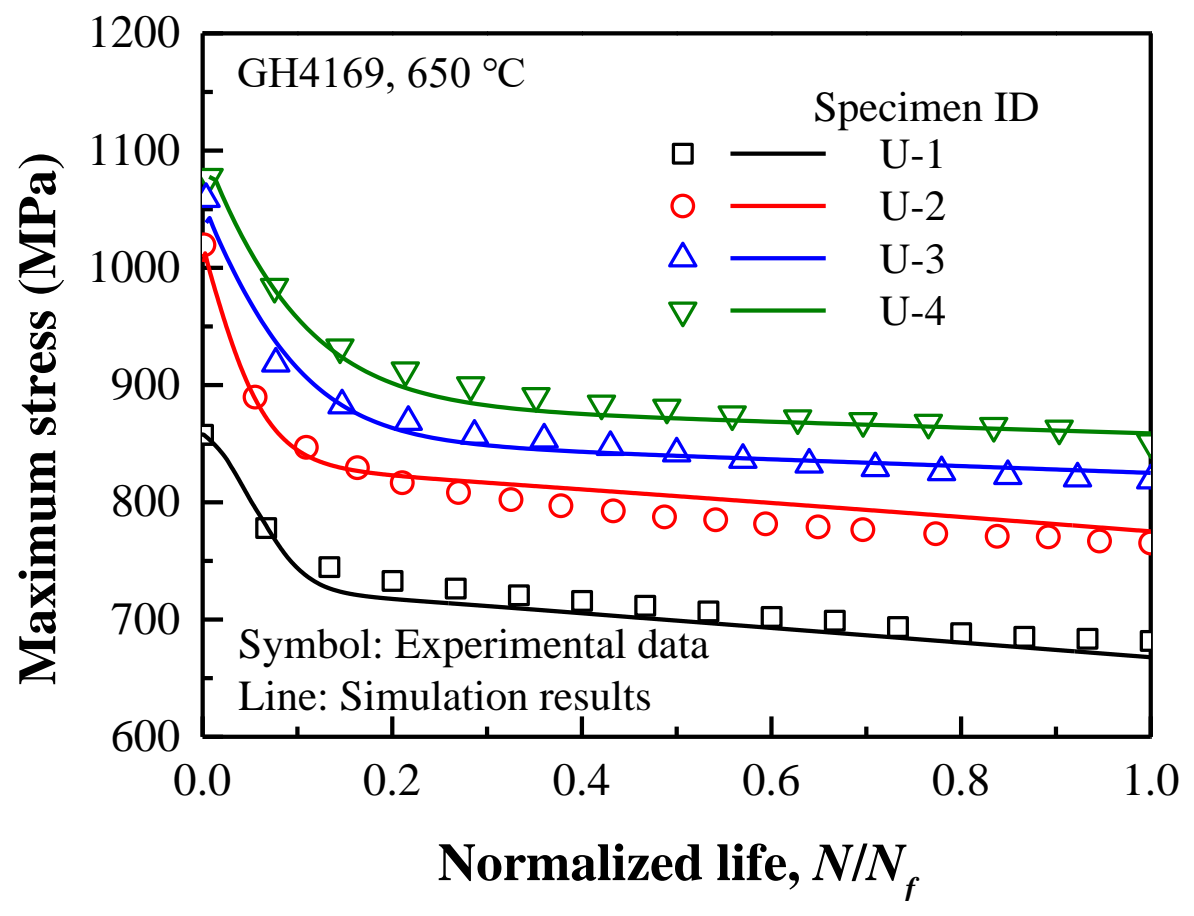

Figure 10 Experimental data and simulation results of cyclic softening curves for specimens $\mathrm{U}-1$ to $\mathrm{U}-4$. 


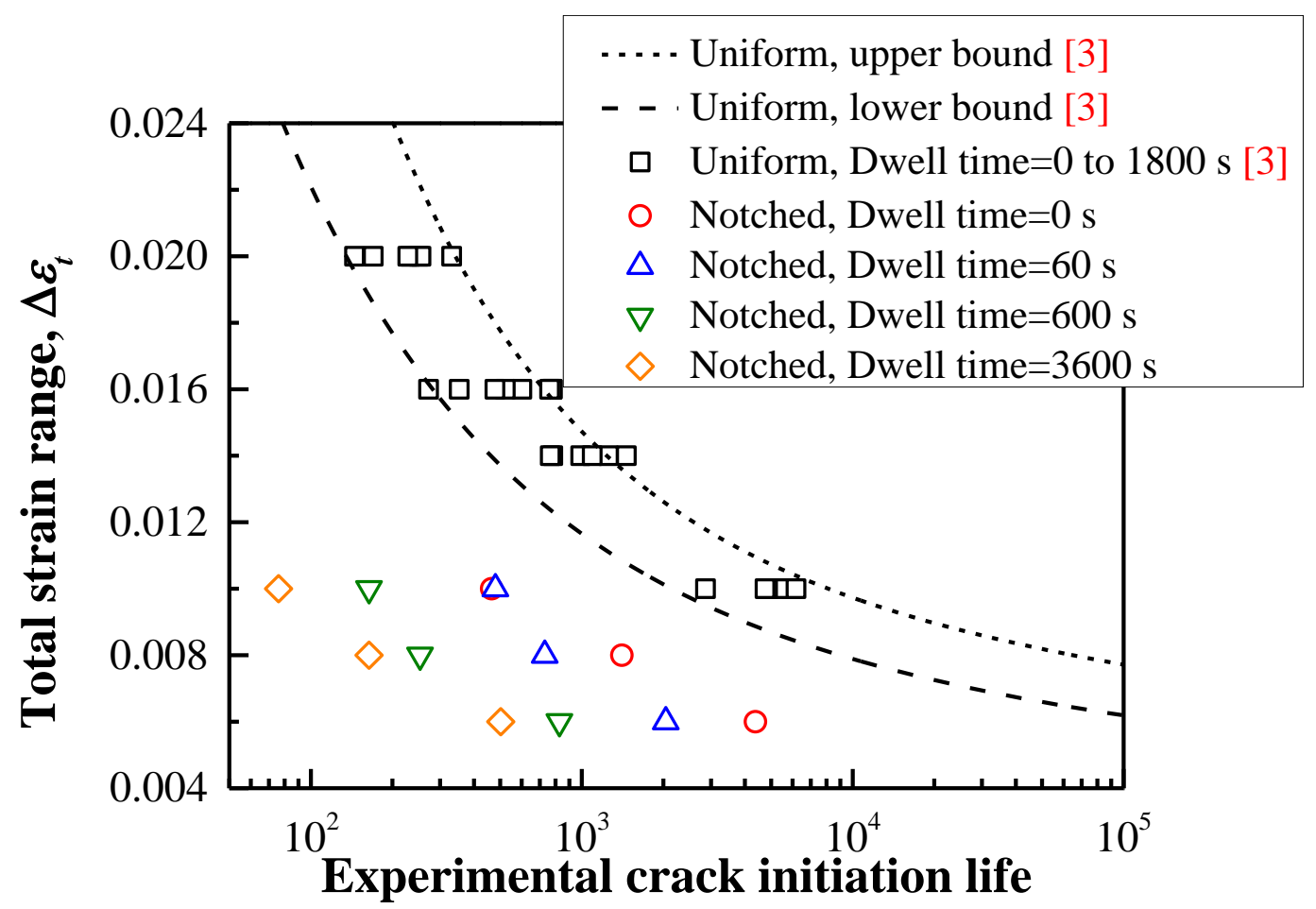

Figure 11 Experimental crack initiation life distributions of uniaixal uniform and notched specimens under creep-fatigue loading waveforms for $\mathrm{GH} 4169$ at $650{ }^{\circ} \mathrm{C}$. 

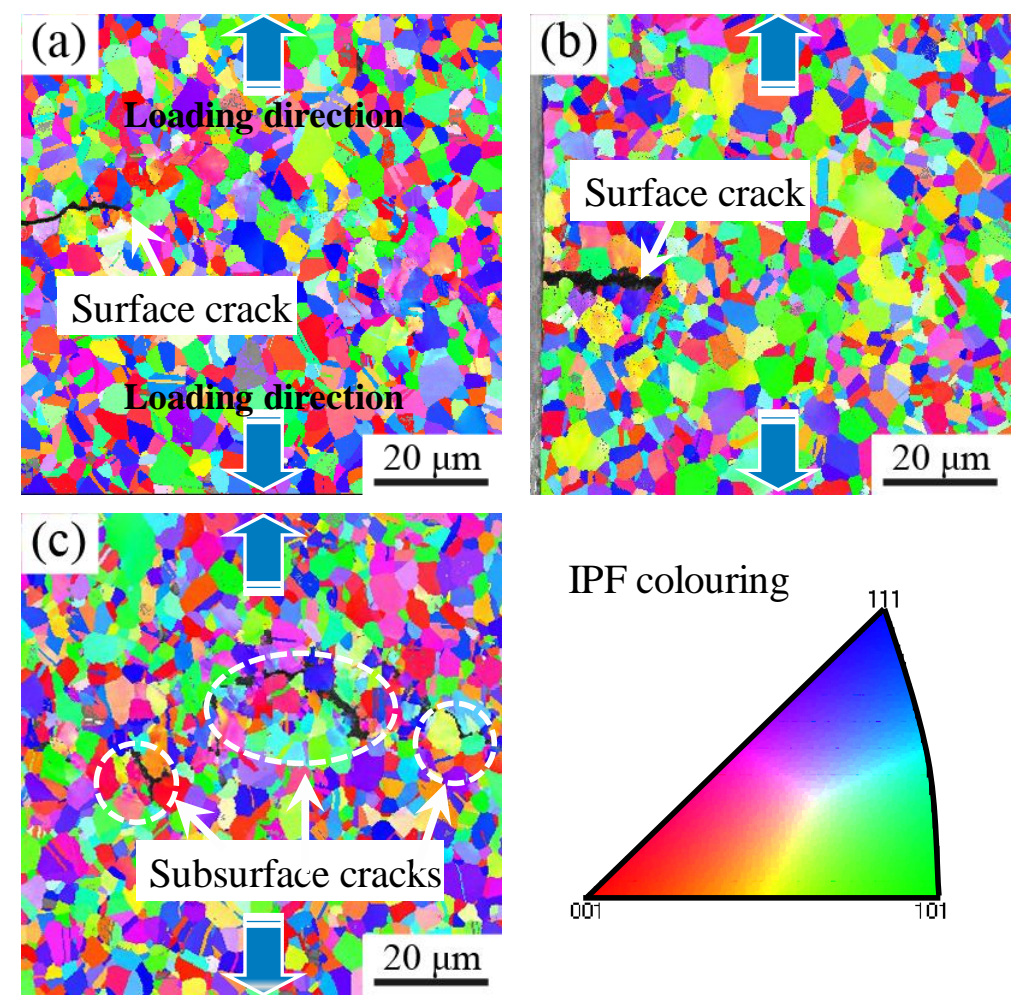

Figure 12 EBSD maps of main crack initiation for (a) specimen $\mathrm{N}-9\left(\Delta \varepsilon_{t}=1.0 \%\right.$, $\left.t_{h}=0 \mathrm{~s}\right),(\mathrm{b})$ specimen $\mathrm{N}-10\left(\Delta \varepsilon_{t}=1.0 \%, t_{h}=60 \mathrm{~s}\right)$, and (c) specimen $\mathrm{N}-12$ $\left(\Delta \varepsilon_{t}=1.0 \%, t_{h}=3600 \mathrm{~s}\right)$. 
(a)
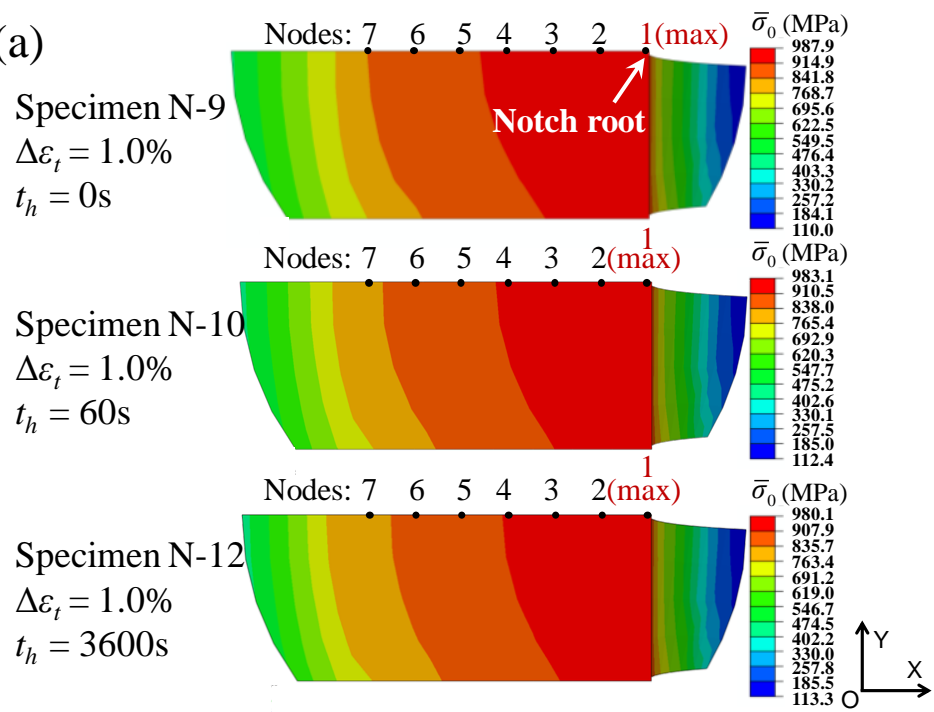

(b)
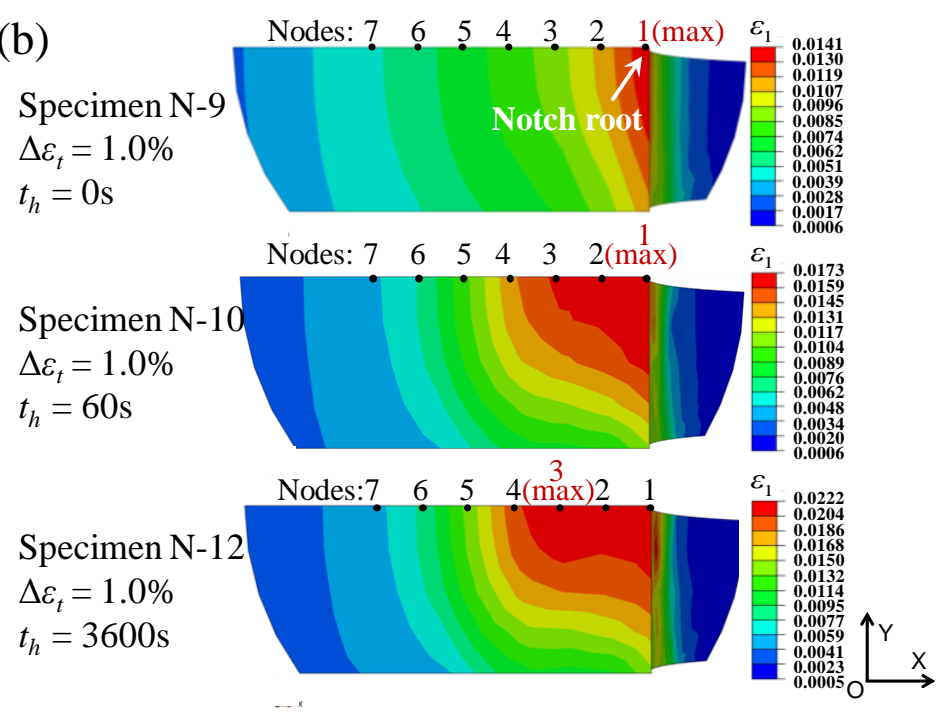

Figure 13 The contours at XOY plane of (a) equivalent peak tensile stress, $\bar{\sigma}_{0}$, and (b) maximum principal strain after hold time period, $\varepsilon_{1}$ at the $100^{\text {th }}$ cycle for specimen N-9 $\left(\Delta \varepsilon_{t}=1.0 \%, t_{h}=0 \mathrm{~s}\right)$, specimen N-10 $\left(\Delta \varepsilon_{t}=1.0 \%, t_{h}=60 \mathrm{~s}\right)$ and specimen $\mathrm{N}-12\left(\Delta \varepsilon_{t}=1.0 \%, t_{h}=3600 \mathrm{~s}\right)$ 

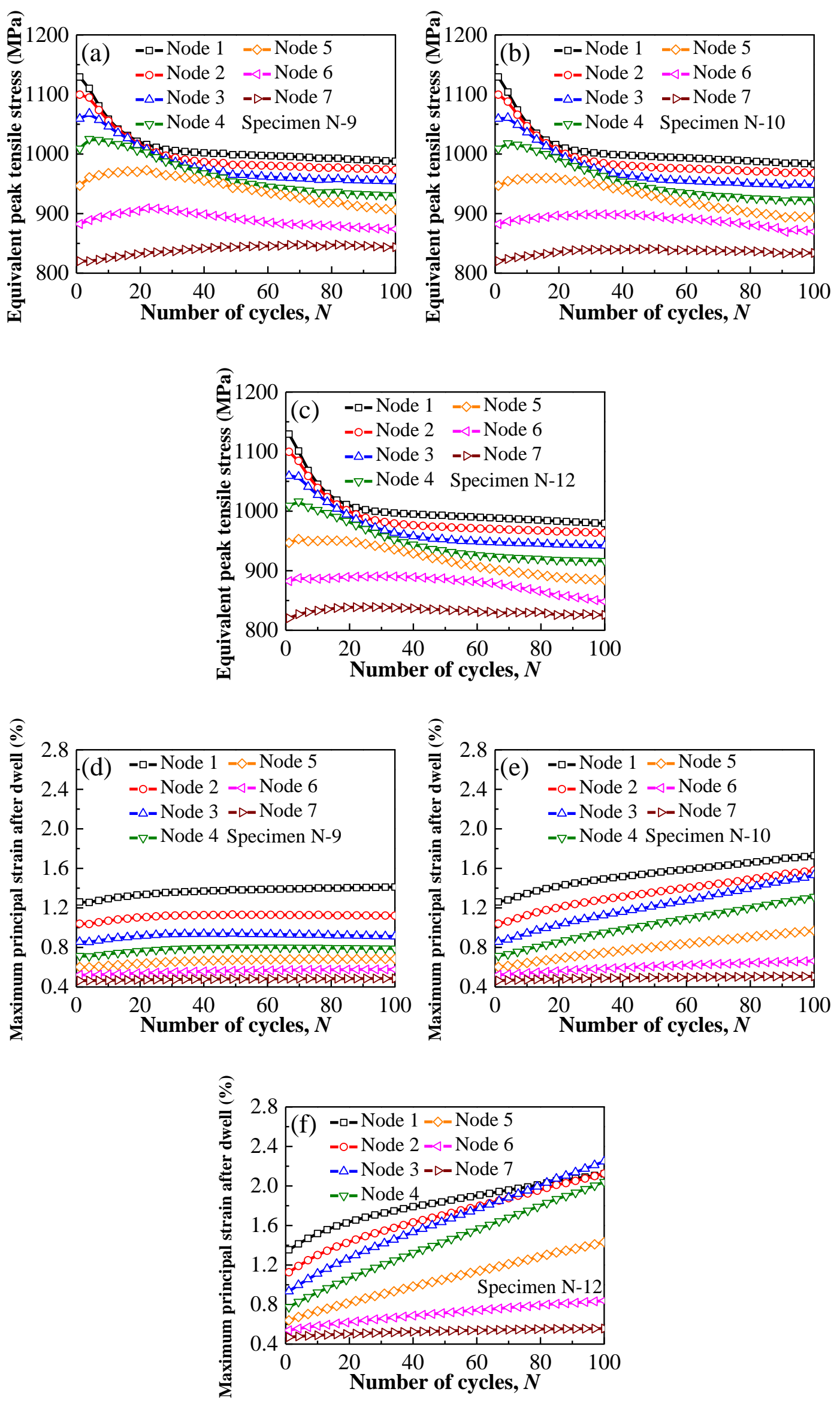
Figure 14 (a) The relations between equivalent tensile peak stress and number of cycles at various nodes for (a) specimen $\mathrm{N}-9\left(\Delta \varepsilon_{t}=1.0 \%, t_{h}=0 \mathrm{~s}\right)$, (b) specimen $\mathrm{N}-10\left(\Delta \varepsilon_{t}=1.0 \%, t_{h}=60 \mathrm{~s}\right)$, and (c) specimen N-12 $\left(\Delta \varepsilon_{t}=1.0 \%, t_{h}=3600 \mathrm{~s}\right)$; and the relations between maximum principal strain and number of cycles for (d) specimen $\mathrm{N}-9$, (e) specimen $\mathrm{N}-10$, and (f) specimen $\mathrm{N}-12$. 
(a)

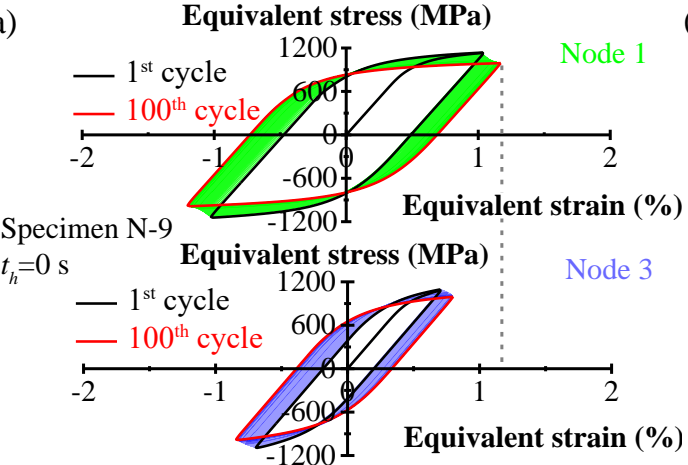

(b)

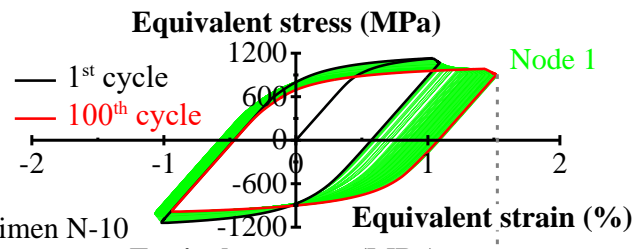

Specimen $\mathrm{N}-10$

Equivalent stress (MPa)

Node 3

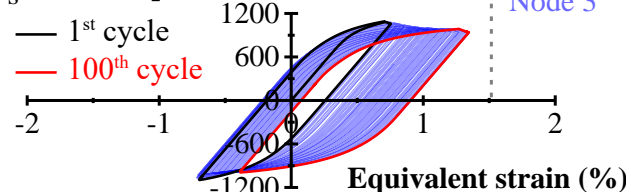

(c)

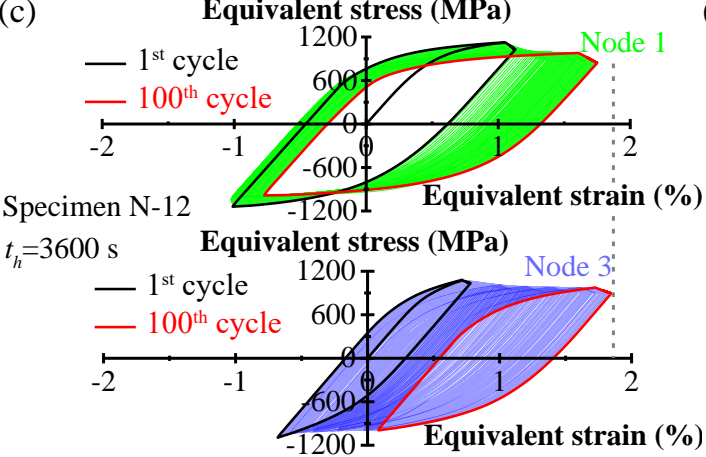

(d)

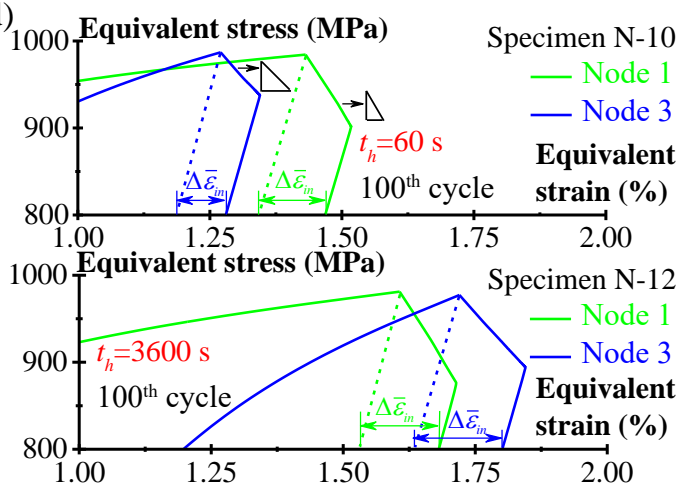

Figure 15 Vertical panel figures of cycle-dependent hysteresis loops at nodes 1 and 3 for (a) specimen N-9 $\left(\Delta \varepsilon_{t}=1.0 \%, t_{h}=0 \mathrm{~s}\right)$, (b) specimen $\mathrm{N}-10\left(\Delta \varepsilon_{t}=1.0 \%\right.$, $\left.t_{h}=60 \mathrm{~s}\right)(\mathrm{c})$ specimen $\mathrm{N}-12\left(\Delta \varepsilon_{t}=1.0 \%, t_{h}=3600 \mathrm{~s}\right)$, and (d) the enlarged view of stress-strain behavior during hold time period at node 1 and 3 of the $100^{\text {th }}$ cycle for specimen $\mathrm{N}-10$ and specimen $\mathrm{N}-12$. 


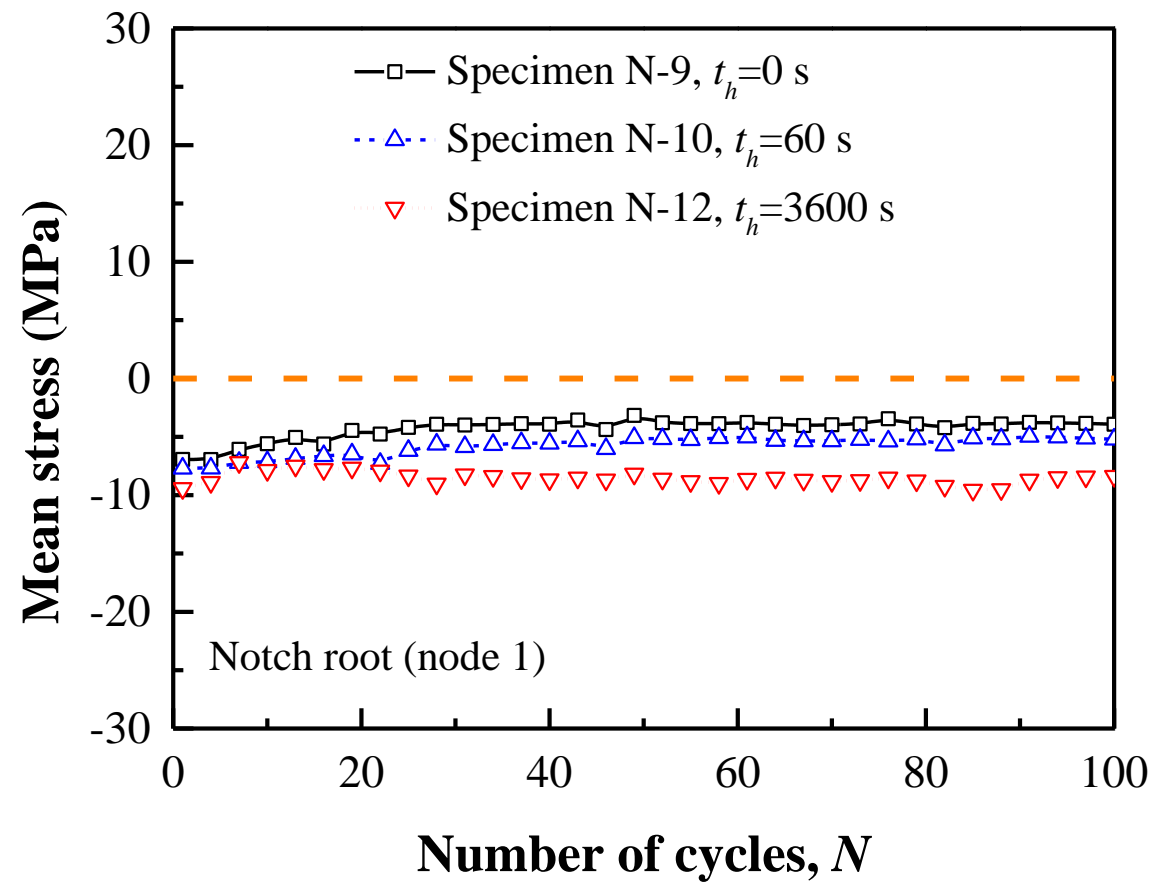

Figure 16 The evolution of mean stress with various numbers of cycles at node 1 of notch root for specimen N-9 $\left(\Delta \varepsilon_{t}=1.0 \%, t_{h}=0 \mathrm{~s}\right)$, specimen $\mathrm{N}-10\left(\Delta \varepsilon_{t}=1.0 \%\right.$, $\left.t_{h}=60 \mathrm{~s}\right)$, and specimen $\mathrm{N}-12\left(\Delta \varepsilon_{t}=1.0 \%, t_{h}=3600 \mathrm{~s}\right)$. 

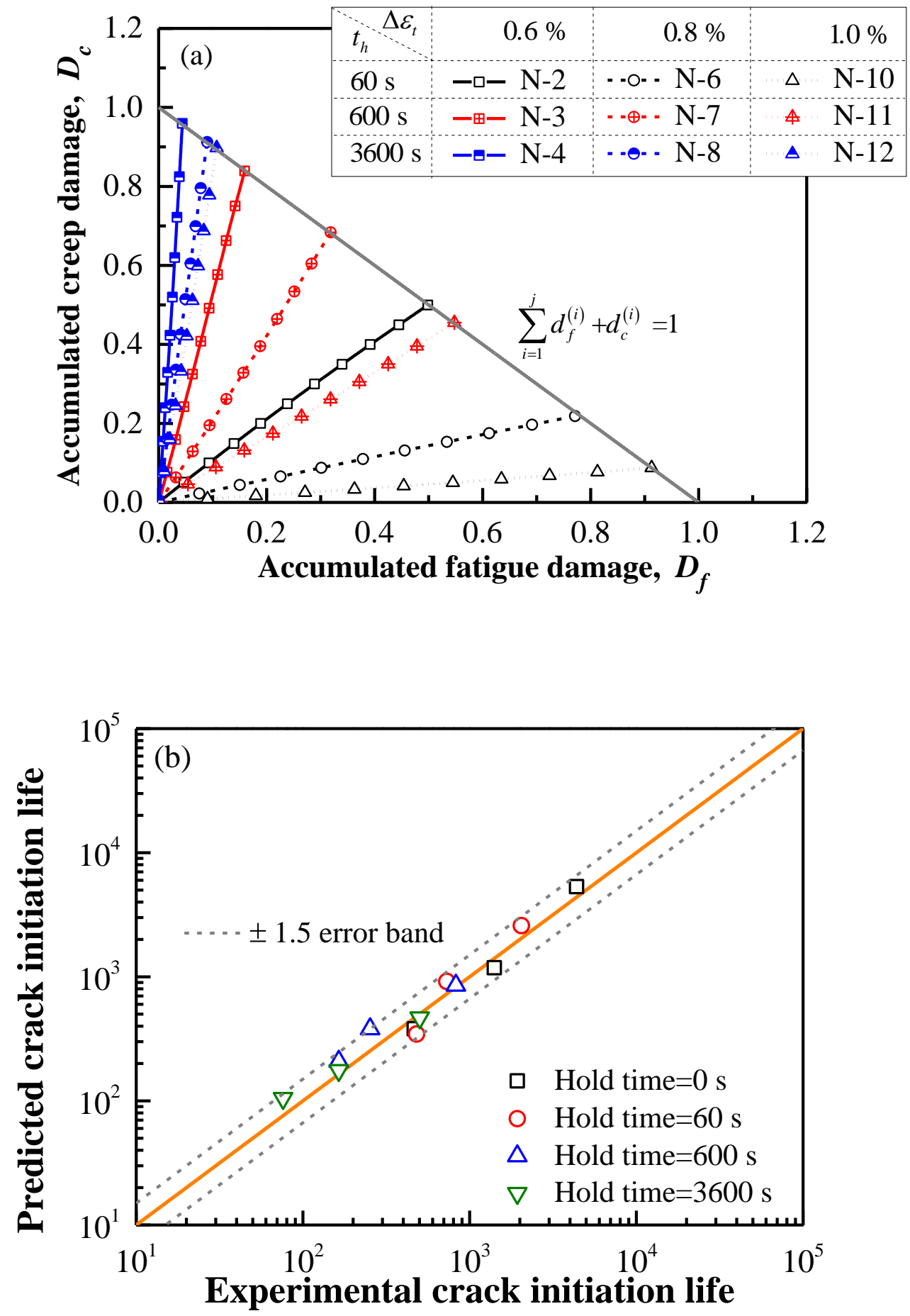

Figure 17 (a) The trajectories for cyclic damage evolutions in the creep-fatigue interaction diagram, and (b) the prediction capacities by using the damage models. 


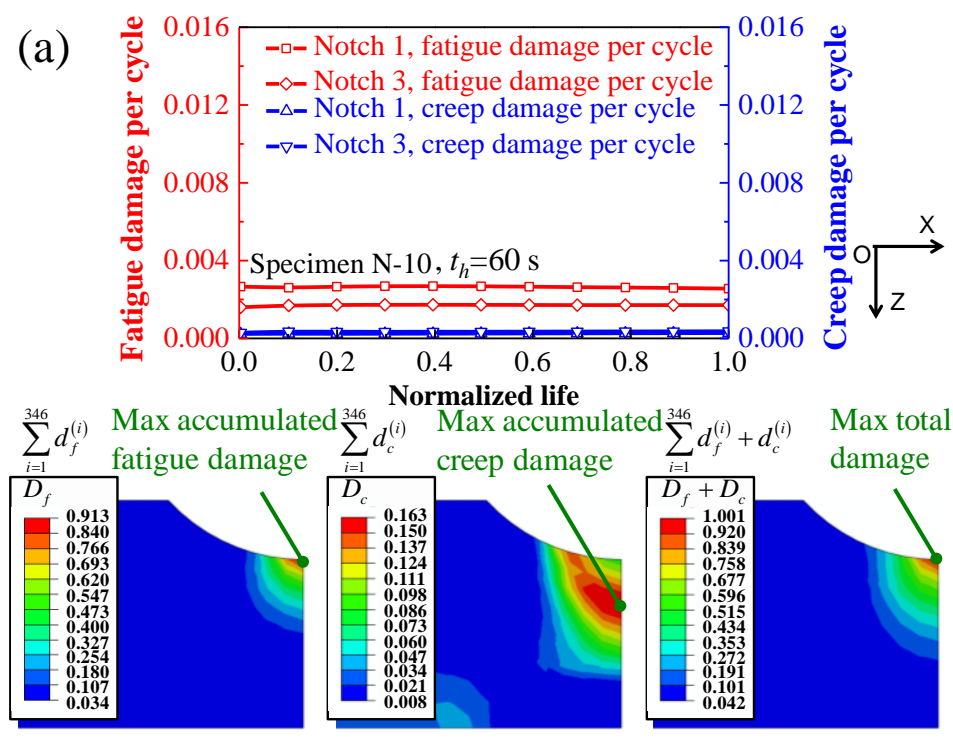

(b) 0.016
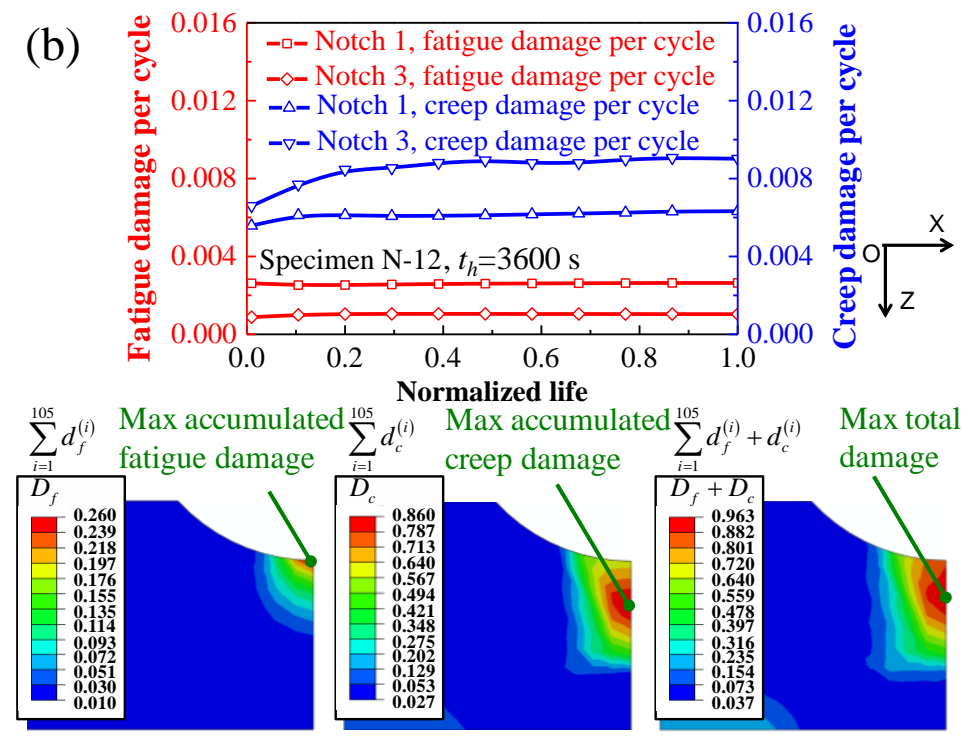

Figure 18 The relations of fatigue/creep damage per cycle and normalized life and corresponding accumulated damage contours at $\mathrm{XOZ}$ plane at the last cycle before crack initiation for (a) specimen N-10 $\left(\Delta \varepsilon_{t}=1.0 \%, t_{h}=60 \mathrm{~s}\right)$ and (b) specimen N$12\left(\Delta \varepsilon_{t}=1.0 \%, t_{h}=3600 \mathrm{~s}\right)$. 


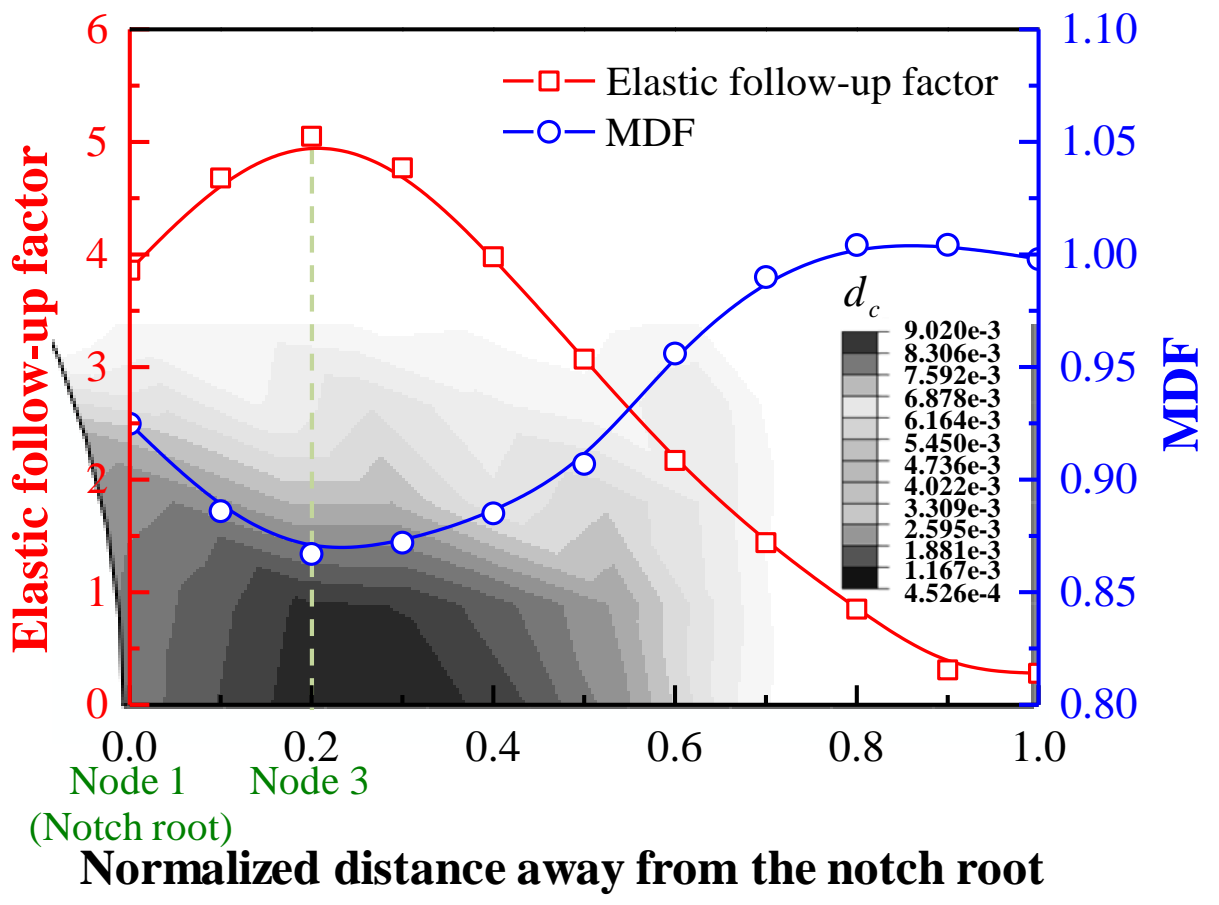

Figure 19 The relations of elastic follow-up factor and the $M D F$ against normalized distance away from the notch root along the top line for specimen $\mathrm{N}-12$ $\left(\Delta \varepsilon_{t}=1.0 \%, t_{h}=3600 \mathrm{~s}\right)$. 


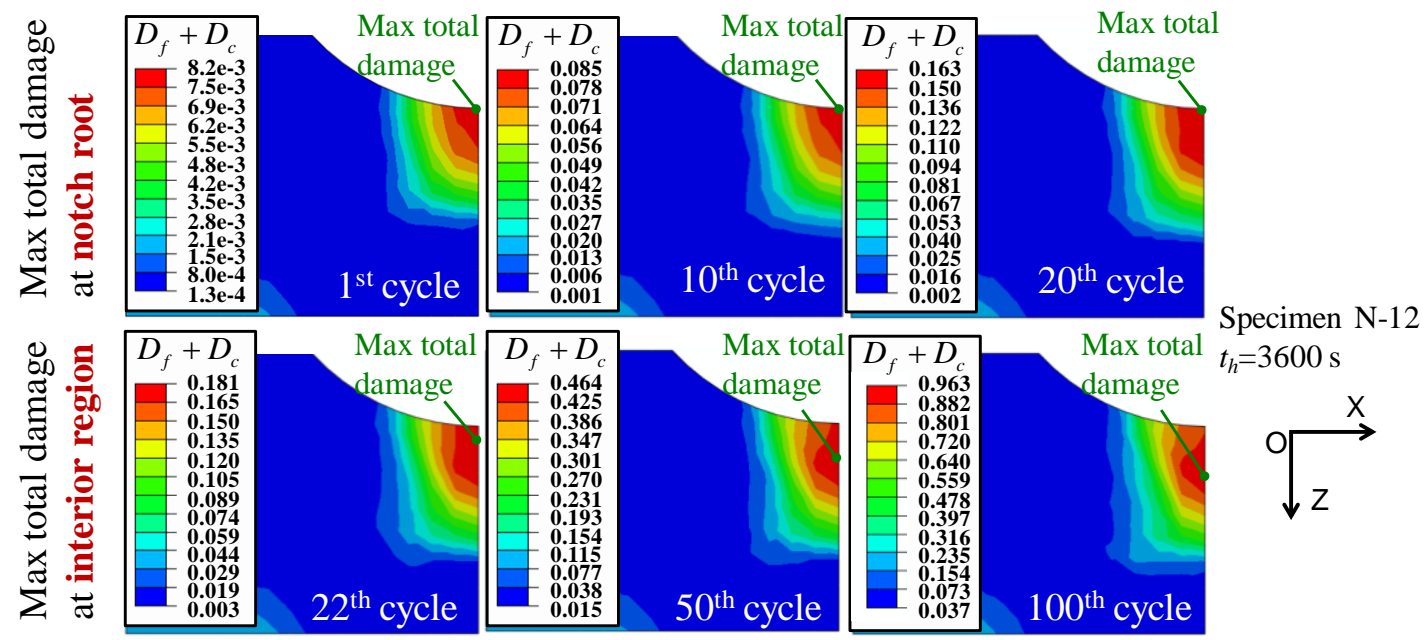

Figure 20 The shifting of most dangerous position with increasing number of cycles for specimen $\mathrm{N}-12\left(\Delta \varepsilon_{t}=1.0 \%, t_{h}=3600 \mathrm{~s}\right)$. 
Table 1 Chemical compositions (wt.\%) of the nickel-based GH4169 superalloy

\begin{tabular}{llllllllllll}
\hline $\mathrm{C}$ & $\mathrm{Mn}$ & $\mathrm{Fe}$ & $\mathrm{S}$ & $\mathrm{P}$ & $\mathrm{Cr}$ & $\mathrm{Mo}$ & $\mathrm{Nb}$ & $\mathrm{Ti}$ & $\mathrm{Al}$ & $\mathrm{B}$ & $\mathrm{Ni}$ \\
\hline 0.023 & 0.03 & 18.75 & 0.001 & 0.012 & 17.86 & 2.98 & 5.38 & 0.99 & 0.57 & 0.0048 & $\mathrm{Bal}$. \\
\hline
\end{tabular}


Table 2 Summary of input parameters for uniaxial and notched specimens in cyclic tests.

\begin{tabular}{llllllc}
\hline Specimen ID & Temp. $\left({ }^{\circ} \mathrm{C}\right)$ & $R_{\varepsilon}$ & $\dot{\varepsilon}(\% / \mathrm{s})$ & $\Delta \varepsilon_{t}(\%)$ & $t_{h}(\mathrm{~s})$ & Life (Cycles) \\
\hline $\mathrm{U}-1$ & 650 & -1 & 0.4 & 1.0 & 300 & 2862 \\
$\mathrm{U}-2$ & 650 & -1 & 0.4 & 1.4 & 300 & 825 \\
$\mathrm{U}-3$ & 650 & -1 & 0.4 & 1.6 & 1800 & 353 \\
$\mathrm{U}-4$ & 650 & -1 & 0.4 & 2.0 & 1800 & 145 \\
$\mathrm{~N}-1$ & 650 & -1 & 0.4 & $0.6^{\mathrm{a}}$ & 0 & 4377 \\
$\mathrm{~N}-2$ & 650 & -1 & 0.4 & $0.6^{\mathrm{a}}$ & 60 & 2043 \\
$\mathrm{~N}-3$ & 650 & -1 & 0.4 & $0.6^{\mathrm{a}}$ & 600 & 827 \\
$\mathrm{~N}-4$ & 650 & -1 & 0.4 & $0.6^{\mathrm{a}}$ & 3600 & 502 \\
$\mathrm{~N}-5$ & 650 & -1 & 0.4 & $0.8^{\mathrm{a}}$ & 0 & 1406 \\
$\mathrm{~N}-6$ & 650 & -1 & 0.4 & $0.8^{\mathrm{a}}$ & 60 & 730 \\
$\mathrm{~N}-7$ & 650 & -1 & 0.4 & $0.8^{\mathrm{a}}$ & 600 & 253 \\
$\mathrm{~N}-8$ & 650 & -1 & 0.4 & $0.8^{\mathrm{a}}$ & 3600 & 164 \\
$\mathrm{~N}-9$ & 650 & -1 & 0.4 & $1.0^{\mathrm{a}}$ & 0 & 465 \\
$\mathrm{~N}-10$ & 650 & -1 & 0.4 & $1.0^{\mathrm{a}}$ & 60 & 480 \\
$\mathrm{~N}-11$ & 650 & -1 & 0.4 & $1.0^{\mathrm{a}}$ & 600 & 164 \\
$\mathrm{~N}-12$ & 650 & -1 & 0.4 & $1.0^{\mathrm{a}}$ & 3600 & 76 \\
\hline
\end{tabular}

${ }^{a}$ Global strain-controlled loading condition within a gauge-length area of $25 \mathrm{~mm}$. 
Table 3 Material constants used in the unified viscoplastic constitutive model for GH4169 superalloy at $650{ }^{\circ} \mathrm{C}$.

\begin{tabular}{l|llll}
\hline Elastic & $E=177 \mathrm{GPa}$ & $v=0.33$ & $Q_{0}=815 \mathrm{MPa}$ & \\
Viscoplastic & $K=400$ & $n=2.0$ & & \\
Kinematic hardening & $\zeta_{1}=6130$ & $\zeta_{2}=1807$ & $\zeta_{3}=892$ & $\zeta_{4}=352$ \\
& $\zeta_{5}=150$ & $\zeta_{6}=88.2$ & $\zeta_{7}=75.0$ & $\zeta_{8}=28.4$ \\
& $r_{1}=23.4$ & $r_{2}=68.0$ & $r_{3}=75.9$ & $r_{4}=48.0$ \\
& $r_{5}=43.4$ & $r_{6}=25.4$ & $r_{7}=54.5$ & $r_{8}=28.0$ \\
Isotropic hardening & $\gamma=4.0 \mathrm{E}-7$ & $\phi_{1}=0.37$ & $\phi_{2}=2.82$ & $\omega=6.6 \mathrm{E}-4$ \\
\hline
\end{tabular}


Table 4 Material constants used in the fatigue damage model based on CPM and creep damage based on modified SEDE for GH4169 superalloy at $650{ }^{\circ} \mathrm{C}$.

\begin{tabular}{|c|c|c|c|c|c|c|c|c|c|c|c|}
\hline \multicolumn{6}{|c|}{ Fatigue } & \multicolumn{6}{|c|}{ Creep } \\
\hline$\tau_{f}^{\prime}$ & $\sigma_{f}^{\prime}$ & $\gamma_{f}^{\prime}$ & $b_{0}$ & $c_{0}$ & $G$ & $\varphi_{1}$ & $n_{1}$ & $n_{2}$ & $A$ & $B$ & $w_{f, \text { trans }}$ \\
\hline 852 & 1476 & 0.28 & -0.086 & -0.58 & 66.5 & 115 & 0.14 & 5.77 & 13.3 & 17.4 & 46.0 \\
\hline
\end{tabular}


Table 5 Mesh independency analysis for specimen N-10 $\left(\Delta \varepsilon_{t}=1.0 \%, t_{h}=60 \mathrm{~s}\right.$, Table 2).

\begin{tabular}{llll}
\hline Meshing strategy & Fatigue damage & Creep damage & Total damage \\
\hline Global 1.0 & $1.17 \times 10^{-3}$ & $9.11 \times 10^{-5}$ & $1.26 \times 10^{-3}$ \\
Global 0.8 & $1.74 \times 10^{-3}$ & $1.43 \times 10^{-4}$ & $1.88 \times 10^{-3}$ \\
Global 0.6 & $2.12 \times 10^{-3}$ & $1.96 \times 10^{-4}$ & $2.32 \times 10^{-3}$ \\
Global 0.4 & $2.60 \times 10^{-3}$ & $2.21 \times 10^{-4}$ & $2.82 \times 10^{-3}$ \\
Global 0.3 & $2.71 \times 10^{-3}$ & $2.32 \times 10^{-4}$ & $2.94 \times 10^{-3}$ \\
Global 0.2 & $2.76 \times 10^{-3}$ & $2.37 \times 10^{-4}$ & $3.00 \times 10^{-3}$ \\
Gradient 0.3 to 1.0 & $2.66 \times 10^{-3}$ & $2.28 \times 10^{-4}$ & $2.89 \times 10^{-3}$ \\
\hline
\end{tabular}

